\title{
Effect of the Reynolds number on turbulence kinetic energy exchanges in flows with highly variable fluid properties
}

\author{
Dorian Dupuy $^{1}$, Adrien Toutant*1, and Françoise Bataille ${ }^{1}$ \\ ${ }^{1}$ PROMES CNRS, Université de Perpignan Via Domitia, Rambla de la thermodynamique, \\ Tecnosud, 66100 Perpignan, France \\ (Published version: Physics of Fluids 31, 015104 (2019); https://doi.org/10.1063/1.5080769)
}

\begin{abstract}
Spatial and spectral energy exchanges associated with the turbulence kinetic energy per unit mass, or half-trace of the velocity covariance tensor, are studied in an anisothermal low Mach number turbulent channel flow. The temperatures of the two channel walls are $293 \mathrm{~K}$ and $586 \mathrm{~K}$. This generates a strong temperature gradient in the wall-normal direction. The effect of the temperature gradient on the energy exchanges is investigated using two direct numerical simulations of the channel, at the mean friction Reynolds numbers 180 and 395. The temperature gradient creates an asymmetry between the energy exchanges at the hot and cold sides, due to the variations of the local fluid properties and low Reynolds number effects. The low Reynolds number effects are smaller at higher Reynolds number, reducing the asymmetry between the hot and cold sides. We also decomposed the energy exchanges in order to study separately the mean-property terms, as found in the constant-property isothermal case, and the thermal terms, specific to flows with variable fluid properties. The significant thermal terms have a similar effect on the flow. Besides, low Reynolds number effects have a negligible impact on thermal terms and only affect mean-property terms.
\end{abstract}

\section{Introduction}

Energy exchanges are fundamental processes of turbulence which drives the evolution of the different parts of total energy. They represent the transformation of energy between different forms and its transfer between scales and locations. Particularly valuable are the energy exchanges associated with turbulence kinetic energy, strongly connected to the intensity of turbulence. The budget of turbulence kinetic energy describes the production, spatial transfer, interscale transport and dissipation of turbulence kinetic energy. In homogeneous isotropic incompressible turbulence, production occurs at large scales and dissipation at small scales, close to Kolmogorov length scale. In the intermediate range of scales, called inertial subrange, an energy cascade transports the energy towards small scales. These processes are Reynolds-number dependent. Dissipation occurs at smaller scales at higher Reynolds number, separating distinctly the scales of production and dissipation [22]. This canonical description of the energy exchanges can be altered profoundly in more complex flow configuration or if additional physical phenomena are involved, for instance in compressible turbulent reacting flows [63] or in magnetohydrodynamic turbulence $[66,67]$. In this paper, we focus on the turbulence kinetic energy exchanges in strongly

\footnotetext{
${ }^{*}$ Corresponding author : adrien.toutant@univ-perp.fr
} 
anisothermal wall-bounded flows at low Mach number. These flows are found in many industrial applications, including concentrated solar power plants [14, 15, 55, 56, 57, 62], heat exchangers and cooling systems $[73,10,35,74]$ or fluids at supercritical pressure within a small temperature range $[68,52,47]$. They are characterised by a strong influence on the energy exchanges of the walls and of temperature.

Wall-bounded turbulent flows are intrinsically anisotropic and inhomogeneous in the wall-normal direction. This provides a very different physics from homogeneous isotropic turbulence because energy is both transferred spatially and transported in between scales. Near the wall, turbulence is driven by an autonomous cycle, relatively independent of the outer flow [30, 21], and two-way interactions between the structures of the inner and outer layers $[28,17,31,27,41]$. The study of the energy exchanges between the different parts of total energy can give a representation of the exchanges of energy in the wall-normal direction and between scales. Knowledge of the three-dimensional flow fields is required to compute the turbulence kinetic energy exchanges. Direct numerical simulations is an useful tool to obtain such data, although it is computationally expensive because the length and time scales of turbulence are small near the wall [60, 29]. Using direct numerical simulations, the energy exchanges associated with turbulence kinetic energy have been investigated in channel flows for various friction Reynolds number: 180 [33, 46, 1, 25, 64, 38], 395 [46, 1], 550-640 [46, 16, 1, 25], 950-1000 [25, 38], 2000 [24, 25] and 5200 [38]. The spectral turbulence kinetic energy budget has been studied for the friction Reynolds numbers 170-210 [18, 7, 43, 54, 2], 500-615 [43, 37, 54], 900-1000 [43, 37, 2] and 5200 [37]. Andrade et al. [2] showed that the spectral range of energy exchanges is broader at higher Reynolds number due to an increase in the low wavenumber scales. Studies of energy exchanges have also been carried out in more complex wall-bounded geometries [6, 54, 44].

In strongly anisothermal wall-bounded flows, the energy exchanges are also under the influence of the variations of temperature. Contrary to high-speed compressible flows, acoustic waves have a negligible impact in strongly anisothermal low Mach number flows $[49,42,48]$. Velocity and temperature are nevertheless strongly coupled because the temperature gradient leads to significant variations of the fluid properties (density, viscosity and thermal conductivity). The temperature dependence of the fluid properties alters the behaviour of flow and of the energy exchanges [8, 39, 26, 36]. As noted by Serra et al. [57], temperature acts as a strong external agency analogous to a strong magnetic field $[71,69,70]$ or rotation $[72,58,59]$. In particular, the variations of the fluid properties create an asymmetry between the two sides of strongly anisothermal turbulent channel flows $[55,62]$. The study of the effect of the asymmetry on the energy exchanges requires the choice of a decomposition of kinetic energy, as several approaches have been suggested in variable-property flows $[13,5,11,23,11,19]$. Aulery et al. [3, 4] investigated the energy exchanges associated with turbulence kinetic energy in the spectral domain using a weighting by the square-root of density to decompose total energy $[32,12]$. Dupuy et al. [20] analysed the energy exchanges in the spatial and spectral domains using a ternary decomposition of kinetic energy [19]. For a mean friction Reynolds number $R e_{\tau}=180$, the effect of the temperature gradient on the energy exchanges was identified to be in large part the combined effect of the mean local variations of the fluid properties and a Reynolds number effect.

This paper investigates the effect of the Reynolds number on the energy exchanges associated with the half-trace of the velocity covariance tensor using direct numerical simulations of a strongly anisothermal channel flow at the mean friction Reynolds numbers $R e_{\tau}=180$ and $R e_{\tau}=395$. The temperatures of the two channel walls are $293 \mathrm{~K}$ and 


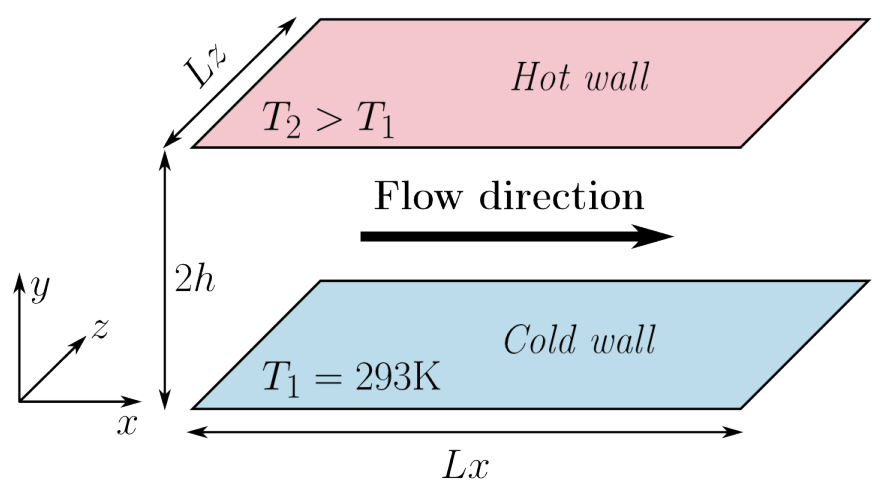

Figure 1 - Biperiodic anisothermal channel flow.

$586 \mathrm{~K}$ for the two simulations. The analysis is carried out in the spatial and spectral domains, using the same approach as Dupuy et al. [20], and focus on the effect of the mean friction Reynolds number on the energy exchanges and on the influence of the temperature gradient. Following Dupuy et al. $[19,20]$, the energy exchanges are decomposed into meanproperty terms, formally identical to the terms present in the constant-property isothermal configuration, and thermal terms, specific to flows with variable fluid properties. The effect of the mean friction Reynolds number on mean-property and thermal terms is compared.

The numerical method and the study configuration is given in section 2 . The equations of the energy exchanges are presented in section 3 . The results are analysed in section 4 .

\section{Study configuration}

\subsection{Channel flow configuration}

Let us consider a flow in an anisothermal biperiodic channel flow (figure 1). The channel is periodic in the streamwise $(x)$ and spanwise $(z)$ directions. There is a strong temperature gradient in the wall-normal direction $(y)$ : the temperature at the cold wall $(y=0)$ is $T_{1}=293 \mathrm{~K}$; the temperature at the hot wall $(y=2 h)$ is $T_{2}=586 \mathrm{~K}$. There is a streamwise volume force in the channel to balance dissipative forces. We study the channel from direct numerical simulations of the low Mach number equations at the mean friction Reynolds numbers $R e_{\tau}=180$ and $R e_{\tau}=395$. Here, the mean friction Reynolds number is the average of the friction Reynolds numbers $R e_{\tau, \omega}=U_{\tau} h / \nu_{\omega}$ at the hot and cold sides, with $\nu_{\omega}$ the kinetic viscosity at the wall and $U_{\tau}=\left[\nu_{\omega}\left(\partial_{y} \bar{U}_{x}\right)_{\omega}\right]^{0.5}$ the friction velocity. The direct numerical simulation at $R e_{\tau}=180$ has been presented in Dupuy et al. [20].

\subsection{Governing equations}

The low Mach number equations are an approximation of the Navier-Stokes equations relevant for flows that are turbulent and strongly anisothermal but slow compared to the speed of sound (Mach number below $10^{-2}$ ). Purely compressible effects of high-speed flows, such as acoustic waves, are neglected using the method of Paolucci [49]. We assume in addition an ideal gas, Newton's law of viscosity and Fourier's law. The flow governing equations are given by: 
- Mass conservation

$$
\frac{\partial \rho}{\partial t}+\frac{\partial \rho U_{j}}{\partial x_{j}}=0
$$

- Momentum conservation

$$
\frac{\partial \rho U_{i}}{\partial t}=-\frac{\partial \rho U_{j} U_{i}}{\partial x_{j}}-\frac{\partial P}{\partial x_{i}}+\frac{\partial \Sigma_{i j}(\boldsymbol{U}, T)}{\partial x_{j}},
$$

- Energy conservation

$$
\frac{\partial U_{j}}{\partial x_{j}}=-\frac{1}{\gamma P_{0}}\left[(\gamma-1) \frac{\partial Q_{j}(T)}{\partial x_{j}}+\frac{\partial P_{0}}{\partial t}\right],
$$

- Ideal gas law

$$
T=\frac{P_{0}}{\rho r},
$$

where $\rho$ is the density, $T$ the temperature, $t$ the temps and $U_{k}$ the $k$-th component of velocity. Einstein summation convention is used and $\delta_{i j}$ is the Kronecker delta. The pressure is decomposed in two terms. The thermodynamical pressure $P_{0}$, constant in space, is the mean pressure in the domain. The mechanical pressure $P$, space-dependent, is related to momentum variations. The shear-stress tensor is given by

$$
\Sigma_{i j}(\boldsymbol{U}, T)=\mu(T)\left[\left(\frac{\partial U_{i}}{\partial x_{j}}+\frac{\partial U_{j}}{\partial x_{i}}\right)-\frac{2}{3} \frac{\partial U_{k}}{\partial x_{k}} \delta_{i j}\right] .
$$

The dynamic viscosity $\mu(T)$ is specified by Sutherland's law [61],

$$
\mu(T)=\mu_{0}\left(\frac{T}{T_{0}}\right)^{\frac{3}{2}} \frac{T_{0}+S_{1}}{T+S_{1}},
$$

using $\mu_{0}=1.716 \cdot 10^{-5} \mathrm{~Pa} \mathrm{~s}, S_{1}=110.4 \mathrm{~K}$ and $T_{0}=273.15 \mathrm{~K}$. The heat flux is given by

$$
Q_{j}(T)=-\lambda(T) \frac{\partial T}{\partial x_{j}} .
$$

The thermal conductivity $\lambda(T)$ is specified assuming a constant Prandtl number, $\lambda(T)=$ $\mu(T) C_{p} / P r$, using $\operatorname{Pr}=0.76$ and $C_{p}=1005 \mathrm{~J} \mathrm{~kg}^{-1} \mathrm{~K}^{-1}$. The ideal gas specific constant is $r=287 \mathrm{~J} \mathrm{~kg}^{-1} \mathrm{~K}^{-1}$. The mean value of the thermodynamic pressure is $\bar{P}_{0}=1.5 \cdot 10^{5} \mathrm{~Pa}$.

\subsection{Numerical settings}

The simulation domain is discretised using a mesh uniform in the streamwise and spanwise directions and uneven in the wall-normal direction. In the wall-normal direction, the mesh follows a hyperbolic tangent law of the form

$$
y_{k}=L_{y}\left(1+\frac{1}{a} \tanh \left[\left(\frac{k-1}{N_{y}-1}-1\right) \tanh ^{-1}(a)\right]\right),
$$

with $a$ the mesh dilatation parameter and $N_{y}$ the number of nodes in the half-height of the channel. The domain size and grid spacing of the simulations are given in table 1 . There is a similar level of refinement at $R e_{\tau}=180$ and $R e_{\tau}=395$. 
Number of grid points Dimension of the domain

$$
N_{x} \times N_{y} \times N_{z}
$$

$$
L_{x} \times L_{y} \times L_{z}
$$

Cell sizes in wall units

DNS180-2

$384 \times 266 \times 384$

$4 \pi h \times 2 h \times 2 \pi h$

$4 \pi h \times 2 h \times(4 / 3) \pi h$

$\Delta_{x}^{+} ; \Delta_{y}^{+}(0)-\Delta_{y}^{+}(h) ; \Delta_{z}^{+}$

DNS395-2 $768 \times 512 \times 512$

Table 1 - Computational domain and cell sizes of the simulations.
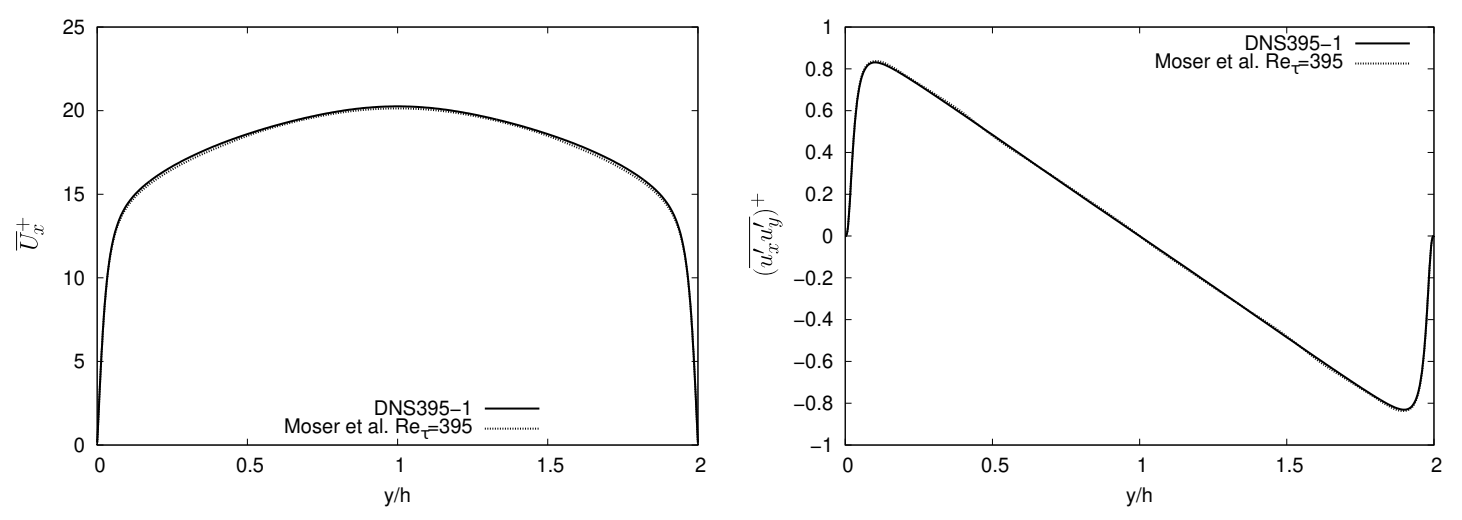

Figure 2 - Validation of the profiles of the mean streamwise velocity $\bar{U}_{x}$ (left) and the covariance of streamwise and wall-normal velocity $\overline{u_{x}^{\prime} u_{y}^{\prime}}$ (right) in the isothermal channel at $R e_{\tau}=395$. The values are scaled by the friction velocity. The reference data is from Moser et al. [46].

We use a finite difference method on a staggered grid $[45,48]$ with a third-order RungeKutta time scheme [65], a fourth-order centred momentum convection scheme. The simulations are performed using the TrioCFD software [9]. To compute the turbulence statistics, a data collection duration of 42 characteristic time $\left(h / U_{\tau}\right)$ is used at $R e_{\tau}=180$ and 40 characteristic time at $R e_{\tau}=395$.

The numerical method has been validated by a mesh convergence study and the comparison of the results in the constant-property isothermal case to the reference data of Moser et al. [46], Vreman and Kuerten [64], Lee and Moser [38], Bolotnov et al. [7] at $R e_{\tau}=180$ and Moser et al. [46] at $R e_{\tau}=395$. The validation of the simulation at $R e_{\tau}=180$ may be found in Dupuy et al. [20]. The validation of turbulence statistics at $R e_{\tau}=395$ is presented in figure 2 in the isothermal case. Our results agree with the reference data of Moser et al. [46], validating the numerical method at the constant-property limit.

\section{Equations of the energy exchanges}

We study the terms of the evolution equation of the half-trace of the velocity covariance tensor in the spatial and spectral domain. Denoting $\left(^{-}\right)$the statistical average operator and (') the fluctuating part operator, the half-trace of the velocity covariance tensor is defined as $e=\frac{1}{2} u_{i}^{\prime} u_{i}^{\prime}$ in the spatial domain. Its evolution can formally be expressed as

$$
\frac{\partial \bar{e}}{\partial t}=\bar{\varphi}+\overline{\mathcal{P}}+\bar{\zeta}+\bar{\varepsilon}
$$

where $\bar{\varphi}=\bar{\varphi}^{c}+\bar{\varphi}^{p}+\bar{\varphi}^{\nu}$ is the sum of the conservative energy transfers related to convection, pressure and viscous stresses, $\overline{\mathcal{P}}$ the production, that is interaction with the other parts of 
kinetic energy, $\bar{\zeta}=\bar{\zeta}^{c}+\bar{\zeta}^{p}+\bar{\zeta}^{\nu}$ the interaction with variable-density kinetic energy and $\bar{\varepsilon}$ the interaction with internal energy. This set of equations is represented schematically on figure 3. It is possible to extend the study to the spectral domain to give the effect of the energy exchanges on the size of turbulent structures. Let us define the hat operator $\left.{ }^{\wedge}\right)$, which gives for any physical quantity $g(x, y, z)$ the coefficients of the Fourier series expansion of $g[40]$ :

$$
\widehat{g}(\boldsymbol{k}, y, t)=\frac{1}{L_{x} L_{z}} \int_{0}^{L_{x}} \int_{0}^{L_{z}} g(\boldsymbol{x}, y, t) \mathrm{e}^{-\mathrm{i} \boldsymbol{k} \cdot \boldsymbol{x}} d \boldsymbol{x},
$$

where $\boldsymbol{x}=(x, z)$ is the position vector in the $x O z$ plane and $\boldsymbol{k}=\left(k_{x}, k_{z}\right)$ the position vector in the $k_{x} O k_{z}$ plane. The spectral counterpart of $e$ is $\check{e}=\frac{1}{2} \widehat{u_{i}^{\prime}} \widehat{u}_{i}^{\prime}$. Its evolution equation can formally be expressed as

$$
\frac{\partial \overline{\check{e}}}{\partial t}=\overline{\check{\varphi}}+\overline{\mathcal{P}}+\overline{\check{\zeta}}+\overline{\check{\varepsilon}}+\bar{\Xi} .
$$

Each term of the spatial decomposition is extended to the spectral domain. In addition, a purely spectral term interscale transport term $\bar{\Xi}$ redistributes the energy between scales without spatial contribution. The spectral energy exchanges are given by:

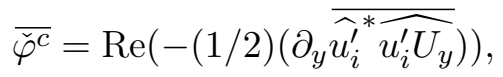

$$
\begin{aligned}
& \overline{\varphi^{p}}=\operatorname{Re}\left(-\partial_{y} \widehat{{\widehat{u_{y}^{\prime}}}^{*} \widehat{P / \rho}}\right) \text {, } \\
& \overline{\breve{\varphi}^{\nu}}=\operatorname{Re}\left(\partial_{y} \widehat{\left.{\widehat{u_{i}^{\prime}}}^{*} \widehat{\Sigma_{i y} / \rho}\right)},\right. \\
& \overline{\mathcal{P}}=\operatorname{Re}\left(-\widehat{u_{i}^{*} \widehat{u_{y}^{\prime}}}\left(\partial_{y} \bar{U}_{i}\right)\right) \text {, } \\
& \bar{\Xi}=\operatorname{Re}\left((1 / 2)\left(\widehat{\widehat{\partial_{j} u_{i}^{\prime}}} * \widehat{u_{i}^{\prime} u_{j}^{\prime}}\right)-(1 / 2)\left(\widehat{{\widehat{u_{i}^{\prime}}}^{*} \widehat{u_{j}^{\prime} \partial_{j} u_{i}^{\prime}}}\right)\right) \text {, } \\
& \check{\zeta} c=\operatorname{Re}\left((1 / 2)\left(\widehat{{\widehat{u_{i}^{\prime}}}^{*} \widehat{u_{i}^{\prime} \partial_{j} U_{j}}}\right)\right) \text {, } \\
& \check{\check{\zeta}^{p}}=\operatorname{Re}\left(-\overline{\left.{\widehat{u_{i}^{\prime}}}^{*} \overline{\left(P / \rho^{2}\right)\left(\partial_{i} \rho\right)}\right)}\right. \text {, } \\
& \check{\check{\zeta}^{\nu}}=\operatorname{Re}\left(\widehat{\left.\widehat{u}_{i}^{\prime} * \overline{\left(\sum_{i j} / \rho^{2}\right)\left(\partial_{j} \rho\right)}\right)},\right. \\
& \bar{\varepsilon}=\operatorname{Re}\left(-\widehat{\widehat{\partial j}_{j}^{\prime}} * \widehat{\Upsilon_{i j} / \rho}\right) .
\end{aligned}
$$

These expressions take into account the symmetries of the geometry $\left(\partial_{x}{ }^{-}=\partial_{z}{ }^{-}=0\right.$ and $\left.\bar{U}_{z}=0\right)$. The spatial terms can be obtained from the integration of the spectral terms on the full wavenumber space. In particular, for any $a$ and $b$, a spectral term of the form $\operatorname{Re}\left(\overline{\widehat{a}^{*} \vec{b}}\right)$ is associated with a spatial term $\overline{a b}$.

In both the spatial and spectral domains, the energy exchanges are decomposed to separate the terms specific variable-property flows (thermal terms, subscript $\Gamma$ ) and terms that are non-zero in the constant-property isothermal case (mean-property terms, subscript $I$ ). The mean-property terms may explicitly depend on the local value of the mean fluid properties outside any derivative. The decomposition is only relevant for the production $\breve{\mathcal{P}}$, the conservative energy transfer $\bar{\varphi}$ and the interaction with internal energy $\bar{\varepsilon}$, which are composed of at least one non-zero mean-property term. Namely, we let $\bar{\varphi}_{I}=\overline{\check{\varphi}_{I}}+\bar{\varphi}_{\Gamma}$, $\overline{\widetilde{\mathcal{P}}}=\overline{\widetilde{\mathcal{P}}_{I}}+\overline{\check{\mathcal{P}}_{\Gamma}}$ and $\bar{\varepsilon}=\overline{\check{\varepsilon}_{I}}+\overline{\varepsilon_{\Gamma}}$. The spectral mean-property terms are given by:

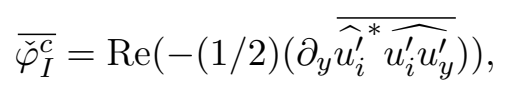




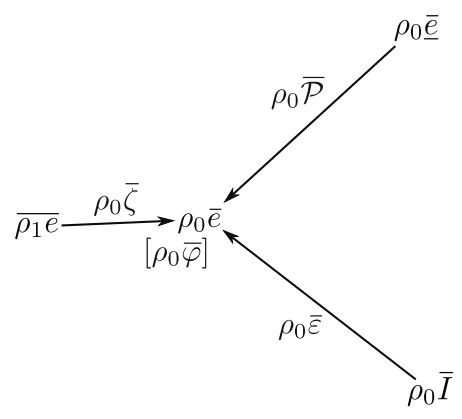

Figure 3 - Schematic representation of the energy exchanges associated with the half-trace of the velocity covariance tensor, up to the constant scalar factor $\rho_{0}$, with $e=\frac{1}{2} u_{i}^{\prime} u_{i}^{\prime}$ the turbulence kinetic energy per unit mass, $\underline{e}=u_{i}^{\prime} \bar{U}_{i}$ the mixed kinetic energy per unit mass and $I$ the internal energy per unit mass $[19,20]$. An arrow represents an interaction between two quantities. This representation follows from the Reynolds decomposition of velocity and the decomposition of density in a constant part $\rho_{0}$ and a variable part $\rho_{1}$.

$$
\begin{aligned}
& \bar{\varphi}_{I}^{p}=\operatorname{Re}\left(-(1 / \bar{\rho})\left(\partial_{y}{\widehat{\widehat{u_{y}^{\prime}}}}^{*} \widehat{P}\right)\right), \\
& \overline{\breve{\varphi}_{I}^{\nu}}=\operatorname{Re}\left(\bar{\nu}\left(\partial_{y} \partial_{y} \overline{\check{e}}\right)+\bar{\nu}\left(\partial_{y} \partial_{y} \widehat{\widehat{u_{y}^{\prime}} \widehat{\widehat{u_{y}^{\prime}}}}\right)\right) \text {, } \\
& \check{\check{\mathcal{P}}_{I}}=\operatorname{Re}\left(-\overline{\widehat{u_{x}^{\prime}}{ }^{*} \widehat{u_{y}^{\prime}}}\left(\partial_{y} \bar{U}_{x}\right)\right) \text {, } \\
& \overline{\varepsilon_{I}}=\operatorname{Re}\left(-2 \bar{\nu}\left({\widehat{\overline{s i j}^{*}}}_{{\overline{s_{i j}^{\prime}}}^{\prime}}\right)\right. \text {. }
\end{aligned}
$$

The interscale transport $\check{\Xi}$ is not decomposed. The thermal energy exchange $\bar{\zeta}$ is zero in the constant-property isothermal case.

\subsection{Wavenumber bin}

The spectral terms are computed using a discrete two-dimensional Fourier transform in the streamwise and spanwise directions,

$$
\begin{aligned}
& \widehat{g}\left(k_{x, m}, y, k_{z, n}\right)= \\
& \frac{1}{N_{x} N_{z}} \sum_{p=0}^{N_{x}-1} \sum_{k=0}^{N_{z}-1} g\left(x_{p}, y, z_{k}\right) \exp \left(-2 \pi \mathrm{i}\left(\frac{m p}{N_{x}}+\frac{k n}{N_{z}}\right)\right) .
\end{aligned}
$$

The analysis of the spectral terms is following Bolotnov et al. [7] based on the use of wavenumber bins. A wavenumber bin is an annulus-shaped portion of the wavenumber space. Each wavenumber vector $\boldsymbol{k}$ is assigned to a wavenumber bin according to its norm $k$ : if $k$ ranges between the lower bound $k_{i}$ and upper bound $k_{i+1}$ of the bin $\# i$, it is assigned to the bin $\# i$. Then, we associate the sum of the values of all wavenumbers assigned to a bin to its wavenumber centre $k_{c, i}$. This procedure removes the spectral directionality of the energy transfers since only the wavenumber norm is taken into account. The same wavenumber bins as in Bolotnov et al. [7] and Dupuy et al. [20] are used:

$$
k_{i}=k_{\min }\left(\frac{k_{\max }}{k_{\min }}\right)^{\frac{i}{N_{b}}},
$$

with $N_{b}=24$ and where $k_{\min }=1 \mathrm{~m}^{-1}$ and $k_{\max }=73 \mathrm{~m}^{-1}$ are the minimum and maximum bound across all bins. The length of the bins is uniform in logarithmic scale. Equal bin 
values therefore represent equal contributions to the integral over the wavenumber space on a logarithmic scale.

\section{Results}

The energy exchanges associated with the half-trace of the velocity covariance tensor are investigated using the semi-local scaling [26], which is an appropriate scaling for the energy exchanges $[50,51,20]$. In contrast with the classical scaling, based only on wall values, the semi-local scaling is based on a velocity scale $U_{\tau}^{*}$ and a length scale $\bar{\nu} / U_{\tau}^{*}$. This leads to a semi-local wall coordinate $y^{*}$, a semi-local wavenumber $k^{*}$, a semi-local velocity $\boldsymbol{U}^{*}$ and a semi-local friction Reynolds number $R e_{\tau}^{*}$,

$$
\begin{aligned}
y^{*} & =\frac{y U_{\tau}^{*}}{\bar{\nu}(y)}, \\
k^{*} & =\frac{k \bar{\nu}(y)}{U_{\tau}^{*}}, \\
\boldsymbol{U}^{*} & =\frac{\boldsymbol{U}}{U_{\tau}^{*}}, \\
R e_{\tau}^{*} & =\frac{U_{\tau}^{*} h}{\bar{\nu}(y)},
\end{aligned}
$$

with $U_{\tau}^{*}$ the semi-local friction velocity, function of the wall shear stress and the local value of the mean density,

$$
U_{\tau}^{*}=\sqrt{\frac{\mu_{\omega}}{\bar{\rho}(y)}\left(\frac{\partial \bar{U}_{x}}{\partial y}\right)_{\omega}},
$$

where the subscript $\omega$ denotes the value at the wall. The terms of the evolution equation of the half-trace of the velocity covariance tensor are scaled by $U_{\tau}^{* 4} / \bar{\nu}(y)$.

The energy exchanges are investigated in the spatial and spectral domains. In both cases, the analysis is carried out in two steps. First, we study the effect of the mean friction Reynolds number on the production, the conservative energy transfer and the interaction with internal energy, through the combined effect of mean-property terms and thermal terms. Then, we study its effect on the mean-property terms and the thermal terms separately.

\subsection{Effect of the Reynolds number in the spatial domain}

\subsubsection{Profiles of the energy exchanges in the anisothermal configuration}

The temperature gradient creates at $R e_{\tau}=180$ and $R e_{\tau}=395$ an asymmetry between the hot and cold sides. The profiles of temperature and of the standard deviation of temperature are provided in figure 4 . The mean and fluctuation profiles are modified by the coupling between turbulence and the temperature gradient (figure 5). This has been investigated in previous studies $[55,56,62,53]$. The temperature gradient also creates an asymmetry between spatial profiles at the hot and cold sides of three energy exchanges associated with the half-trace of the velocity covariance tensor: the production $\overline{\mathcal{P}}$, the conservative energy transfer $\bar{\varphi}$ and the interaction with internal energy $\bar{\varepsilon}$ [20]. The energy exchanges have a greater magnitude at the cold side than at the hot side, but occur over a 

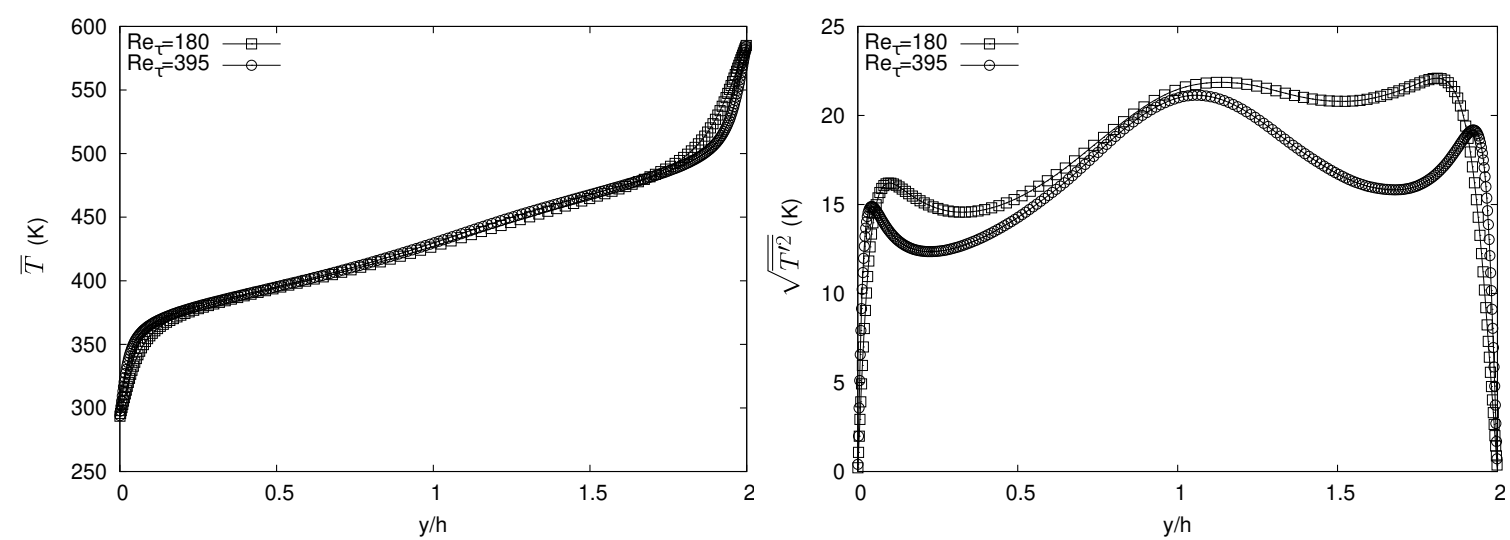

Figure 4 - Profiles of the mean temperature (left) and the standard deviation of temperature (right) without scaling at $R e_{\tau}=180$ and $R e_{\tau}=395$.
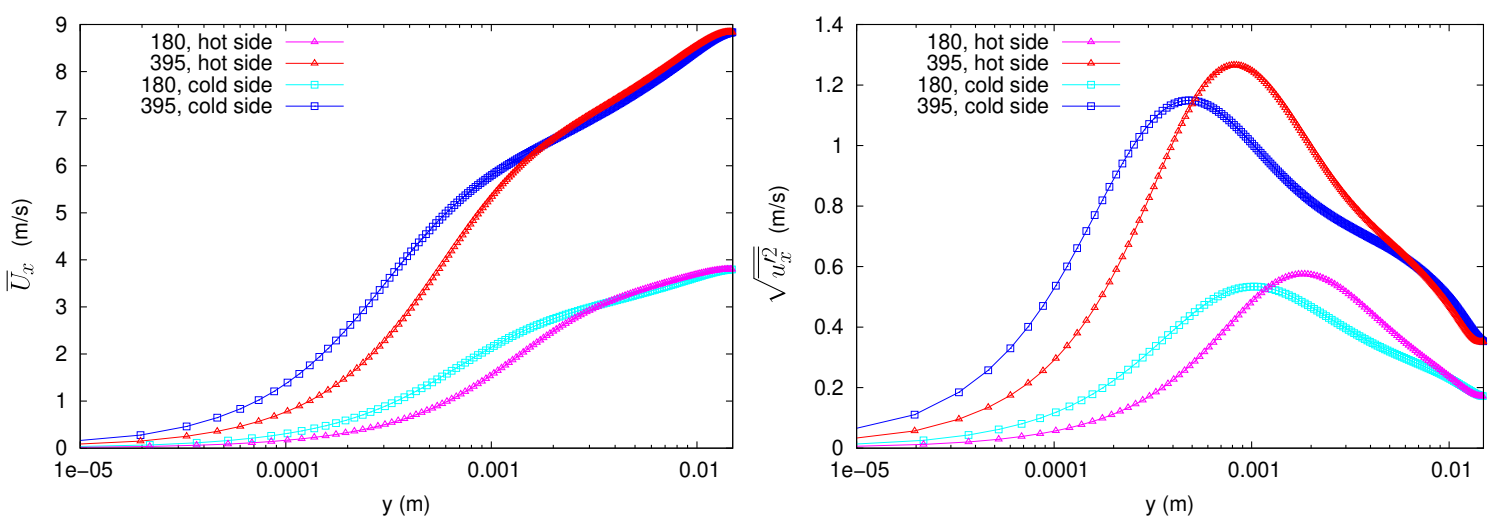

Figure 5 - Profiles of the mean streamwise velocity (left) and the standard deviation of streamwise velocity (right) without scaling at $R e_{\tau}=180$ and $R e_{\tau}=395$.

narrower spatial range and closer to the wall (figure 6). These effects of the temperature gradient are Reynolds number dependent.

The spatial profiles at $R e_{\tau}=180$ and $R e_{\tau}=395$ are provided in figure 7 with the semi-local scaling. The profiles of the two most significant parts of the conservative energy transfer, the viscous transfer and the convection, are also given. At higher mean friction Reynolds number, the asymmetry between the hot and cold sides is reduced. This is consistent with the assumption that the asymmetry between the hot and cold sides is mainly due to two separate contributions [20]:

- the variations of the local velocity and length scales due to the variations of the local fluid properties, to some extent taken into account by the semi-local scaling;

- a varying low Reynolds number effect between the hot and cold sides depending on the semi-local friction Reynolds number (not taken into account by the semi-local scaling).

Indeed, the profiles of the energy exchanges exhibit low Reynolds number effects in the constant-property isothermal case within the Reynolds number range of this study. All energy exchanges investigated have a larger amplitude at the higher Reynolds number with the semi-local scaling (figure 7). This Reynolds number dependence is more pronounced 


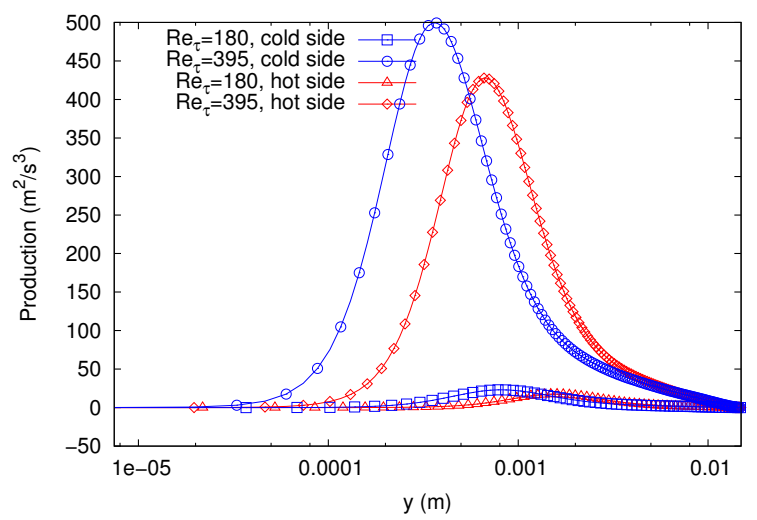

Figure 6 - Profiles of the terms of the production $\overline{\mathcal{P}}$ without scaling at $R e_{\tau}=180$ and $R e_{\tau}$ $=395$.

for the interaction with internal energy and the viscous transfer than for the production and the convection. In addition, the peaks of production (figure 7(a)), of the conservative energy transfer (figure 7(b)), of the viscous transfer (figure 7(d)) and of convection (figure $7(\mathrm{e}))$ are slightly closer to the wall at $R e_{\tau}=395$ than at $R e_{\tau}=180$. In the anisothermal channel, the semi-local friction Reynolds number varies across the channel due to the temperature gradient. At $R e_{\tau}=180$, the semi-local friction Reynolds number ranges from 105 at the hot wall to 260 at the cold wall. At $R e_{\tau}=395$, the semi-local friction Reynolds number ranges from 227 at the hot wall to 556 at the cold wall. The profiles are therefore subjected to a varying low Reynolds number effect between the hot and cold sides, which cannot be expected to be taken into account by any scaling based on the local fluid properties, given that the classical wall scaling does not collapse the constant-property isothermal profiles at various friction Reynolds number.

The semi-local scaling properly accounts for the effect of the variations of the local fluid properties on production at $R e_{\tau}=180$ and $R e_{\tau}=395$ (figure $7(\mathrm{a})$ ). Indeed, the maximum of production with the semi-local scaling follows a low Reynolds number effect according to the empirical relation of Laadhari [34]. While the semi-local scaling is also relevant for the other energy exchanges, the profiles of the conservative energy transfer (figure 7(b)) and the interaction with internal energy (figure 7(c)) show evidence that the approximation of the semi-local scaling does not perfectly take into account the effect of the variations of the local fluid properties. The value of the interaction with internal energy (figure $7(\mathrm{c})$ ) at the wall is not equal to that of the constant-property isothermal profile at the wall semi-local friction Reynolds number. The hot and cold profiles of the viscous transfer (figure $7(\mathrm{~d})$ ) deviates from all constant-property isothermal profiles, even at the point near $y^{*}=3.5$ where they pass through the same points. The asymmetry between the hot and cold profiles of the convection (figure $7(\mathrm{e})$ ) is very small compared to a low Reynolds number effect, especially at the positive extremum, closer from the wall. These results confirms previous findings at $R e_{\tau}=180$ [20].

Hence, the asymmetry between the hot and cold sides is reduced at $R e_{\tau}=395$ compared to $R e_{\tau}=180$ because low Reynolds number effects are smaller the higher the Reynolds number. This only affects one component of the effect of the temperature gradient however. While a simulation at even higher Reynolds number would be free of low Reynolds number effects, the results suggest that the hot and cold profiles would still not collapse because the approximation of the semi-local scaling does not perfectly account for the effect of the 
variations of the local fluid properties. This is investigated further by decomposing the energy exchanges into mean-property terms and thermal terms.

\subsubsection{Profiles of the mean-property terms in the anisothermal configuration}

The spatial profiles with the semi-local scaling of the mean-property terms follows for the most part the same general behaviour as the total energy exchanges and are subject to a similar effect of the Reynolds number (figure 7). The mean-property production is identical to the total production because the thermal production is negligible (figure $7(\mathrm{a})$ ). At $R e_{\tau}=180$, the profiles of the mean-property conservative energy transfer (figure 7(b)) and the mean-property interaction with internal energy (figure $7(\mathrm{c})$ ) at the hot and cold sides are closer to each other and to the constant-property isothermal profiles, especially in an area around $y^{*}=4$ but not at the wall where the mean-property and total terms are identical. At $R e_{\tau}=395$, the effect is similar but goes further, inverting the ordering of the hot and cold profiles at some parts of the channel, leading to a larger amplitude at the hot side. This is due to the reduced asymmetry of the energy exchanges between the hot and cold sides.

\subsubsection{Profiles of the thermal terms in the anisothermal configuration}

We provide in figure 8 the spatial profiles with the semi-local scaling of the three most significant thermal terms at $R e_{\tau}=180$ and $R e_{\tau}=395$ : the thermal conservative energy transfer $\overline{\varphi_{\Gamma}}$ (figure $8($ a)), the interaction with variable density kinetic energy $\bar{\zeta}$ (figure $8(\mathrm{~b})$ ) and the thermal interaction with internal energy $\overline{\varepsilon_{\Gamma}}$ (figure $8(\mathrm{c})$ ). The interaction with variable density kinetic energy is a thermal energy exchange while the thermal conservative energy transfer and the thermal interaction with internal energy are parts of energy exchanges. The thermal production is not discussed because it is negligible compared to the mean-property production. The three investigated thermal terms show some similarity as their profiles are composed of two peaks which are of opposite sign at the hot and cold sides, and have a larger magnitude at the cold side than at the hot side.

The profiles of the thermal terms at the two mean friction Reynolds number are very similar. The effects of the Reynolds number on the thermal terms, very small, are given hereafter. At $R e_{\tau}=395$, the position of the extrema is shifted towards the wall compared to $R e_{\tau}=180$, reducing the position asymmetry between the hot and cold sides for the extremum closer to the centre of the channel. The amplitude of the extremum closer to the wall is increased slightly whereas the amplitude of the extremum closer to the centre of the channel is decreased. These effects are not significant and do not affect notably the amplitude asymmetry between the hot and cold sides. All in all, the Reynolds number dependence of the thermal terms is very slight, suggesting that the profiles are almost universal within the Reynolds number range of this study. Therefore, we may infer the approximate conclusion that low Reynolds number effects only affect the mean-property terms but are negligible on thermal terms. In other words, the thermal terms are functions of the variations of the mean local fluid properties only and do not substantially depend on the Reynolds number.

Since the magnitude of the mean-property terms increases with the mean friction Reynolds number while thermal terms are largely unaffected, the relative contributions of the thermal terms to the energy exchanges decline at high Reynolds number. On the other hand, the relative importance of the thermal terms on the asymmetry between the 


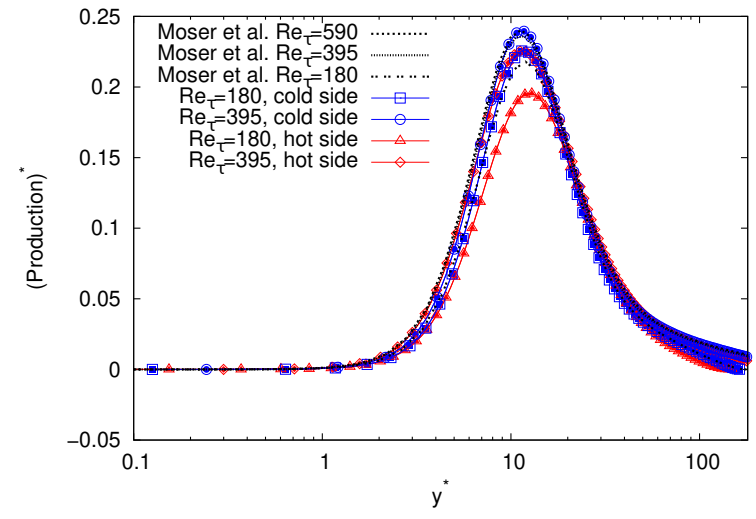

(a) Production, total term $\overline{\mathcal{P}}$ and mean-property term $\overline{\mathcal{P}_{I}}$ (m.-p. term).

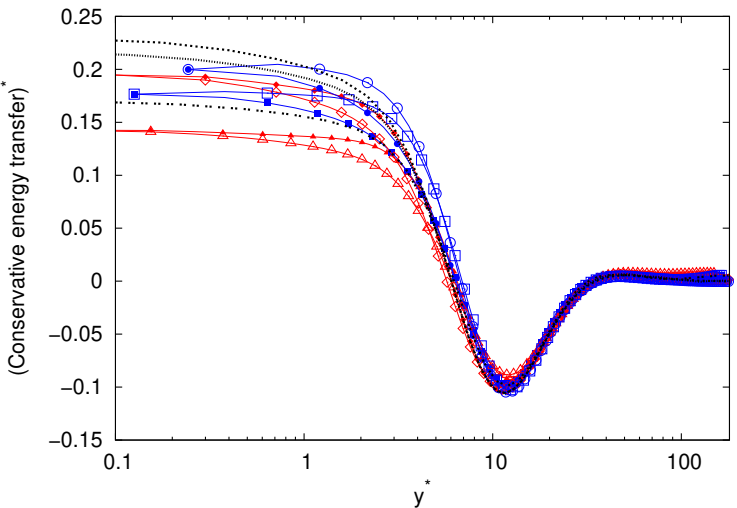

(b) Conservative energy transfer, total term $\bar{\varphi}$ and mean-property term $\overline{\varphi_{I}}$ (m.-p. term).

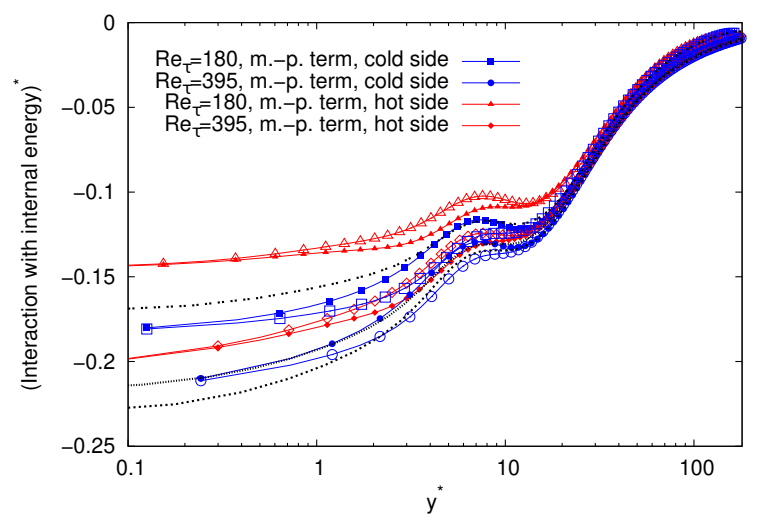

(c) Interaction with internal energy, total term $\bar{\varepsilon}$ and mean-property term $\overline{\varepsilon_{I}^{\nu}}$ (m.-p. term).

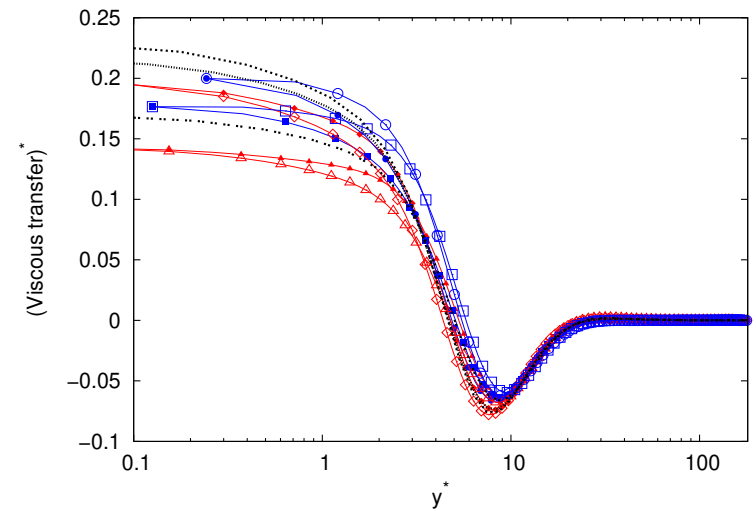

(d) Viscous transfer, total term $\overline{\varphi^{\nu}}$ and meanproperty term $\overline{\varphi_{I}^{\nu}}$ (m.-p. term).

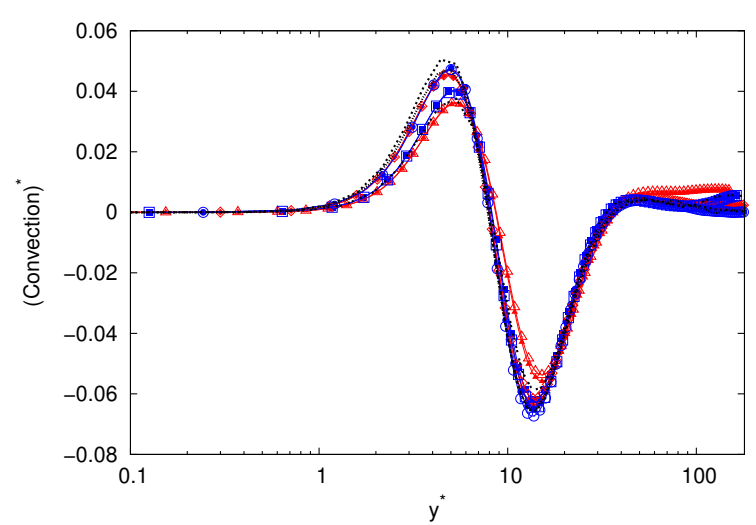

(e) Convection, total term $\overline{\varphi^{c}}$ and mean-property term $\overline{\varphi_{I}^{c}}$ (m.-p. term).

Figure 7 - Profiles of the terms of the budget of the half-trace of the velocity covariance tensor with the semi-local scaling at $R e_{\tau}=180$ and $R e_{\tau}=395$. The profiles of the two most significant parts of the conservative energy transfer are also given. The results are compared to the reference data of Moser et al. [46] for a constant-property isothermal channel flow at the friction Reynolds numbers 180, 395 and 590. The viscous transfer and the convection are the two most significant parts of the conservative energy transfer. 


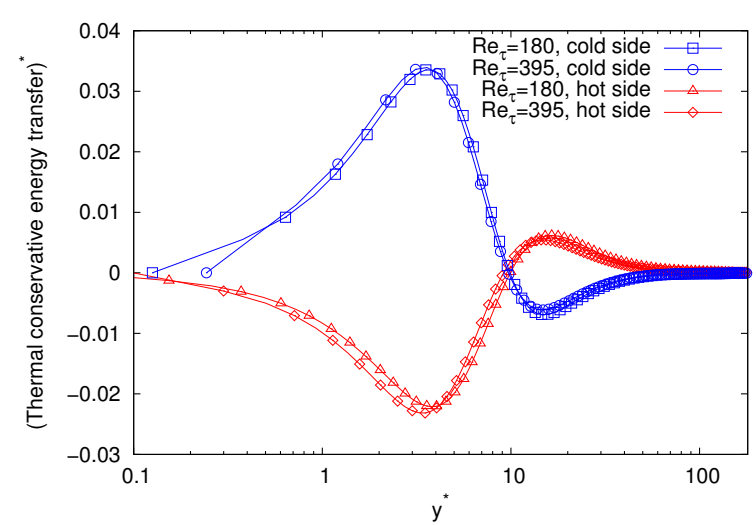

(a) Thermal conservative energy transfer $\overline{\varphi_{\Gamma}}$.

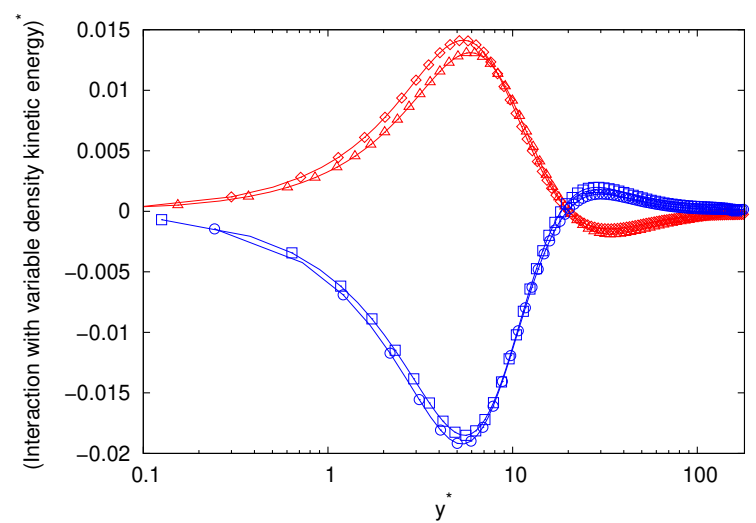

(b) Interaction with variable density kinetic energy $\bar{\zeta}$.

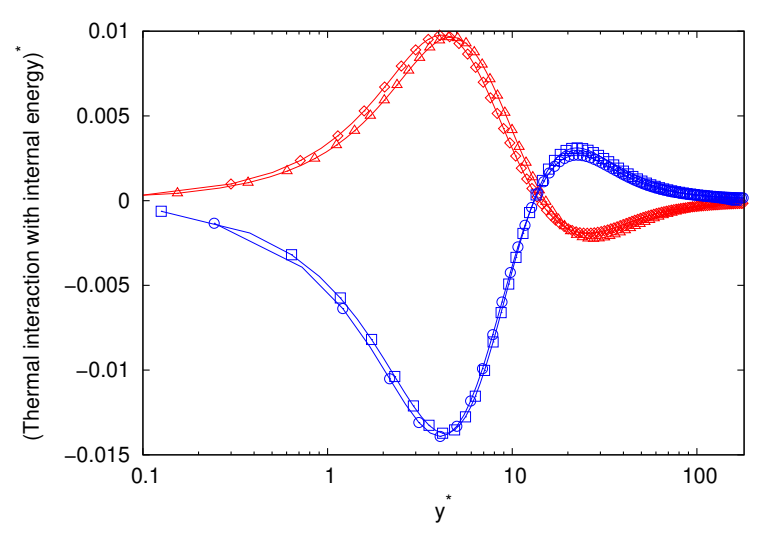

(c) Thermal interaction with internal energy $\overline{\varepsilon_{\Gamma}}$.

Figure 8 - Profiles of the thermal terms of the budget of the half-trace of the velocity covariance tensor with the semi-local scaling at $R e_{\tau}=180$ and $R e_{\tau}=395$.

hot and cold sides become larger, given that the asymmetry between the hot and cold sides is reduced for the mean-property terms. This follows from the assumption that a varying low Reynolds number effect is a significant part of the effect of the temperature gradient on the mean-property terms whereas the thermal terms are only tied to the variations of the mean local fluid properties. Increasing the mean friction Reynolds number reduces the low Reynolds number effects, which gives less weight to the mean-property terms on the asymmetry between the hot and cold sides. However, as noted above, the mean-property terms are also in a large part related to the variations of the local fluid properties since the decomposition does not completely separate the two effects.

\subsection{Effect of the Reynolds number in the spectral domain}

\subsubsection{Spectra of the energy exchanges in the anisothermal configuration}

The effect of the mean friction Reynolds number on the spectral energy exchanges is investigated. The spectral behaviour of the energy exchanges has been described in Dupuy et al. [20] in the constant-property isothermal case and in the anisothermal case. The half-trace of the velocity covariance tensor is in the spectral domain predominantly governed by four spectral energy exchanges: the production $\overline{\mathcal{P}}$, the interscale transport 
$\bar{\Xi}$, the conservative energy transfer $\bar{\varphi}$ and the interaction with internal energy $\bar{\varepsilon}$. The peak production of turbulence kinetic energy occurs around $y^{*}=12$ and $k^{*}=0.07$. The interscale transport redistributes the produced energy among scales, mainly towards small scales but also towards large scales. The energy is, in addition, redistributed spatially by the conservative energy transfer. A pseudo-energy transfer can be identified from the peak production area, where the conservative energy transfer is positive, to the wall and the center of the channel, where the conservative energy transfer is negative. A cut of the spectra at the location of the spatial peak of production, $y^{*} \approx 12$ (figure 9 ) shows that no energy exchange predominates. The produced energy is split almost evenly in three energy processes: its transfer away and towards the wall, its redistribution towards large and small scales and its conversion into internal energy. Near the wall $\left(y^{*}<2\right)$, the production and the purely spectral transfer are negligible. The interaction with internal energy and the conservative energy transfer are thus in balance and occurs at the same wavenumber (figure 10). At the centre of the channel, the production is negligible. Energy coming from the production area is transported towards small scales where it is dissipated. The conservative energy transfer and the interaction with internal energy thus do not occur at the same wavenumber (figure 11). While the Reynolds number differences are addressed by the semi-local scaling at the spatial peak of production or near the wall, the semi-local scaling is not appropriate at the centre of the channel. Since the relevant length scale of turbulence near the centre of the channel is the half-height of the channel $h$ [25], we suggest the use a semi-local outer-flow scaling $\left({ }^{h}\right)$ based on the length scale $h$ and a velocity scale $U_{\tau y}^{*}$ to scale the amplitude of the energy exchanges, with

$$
U_{\tau y}^{*}=\sqrt{\frac{1}{\bar{\rho}(y)}\left[\frac{2 h-y}{2 h} \mu_{1}\left(\frac{\partial \bar{U}_{x}}{\partial y}\right)_{1}-\frac{y}{2 h} \mu_{2}\left(\frac{\partial \bar{U}_{x}}{\partial y}\right)_{2}\right]} .
$$

The linear combination of the wall shear stresses at the hot and cold sides ensures that hot and cold profiles are equal at the centre of the channel but takes into account the variations of the mean friction Reynolds number. With this scaling, the peak of the conservative energy transfer has a smaller semi-local wavenumbers $k^{*}$ at $R e_{\tau}=395$ than at $R e_{\tau}=180$, whereas dissipation occurs at similar semi-local wavenumbers $k^{*}$ (figure 11). Note however that the unscaled wavenumbers of the conservative energy transfer are similar at $R e_{\tau}=395$ and $R e_{\tau}=180$, whereas the scales of dissipation are smaller at high Reynolds number without scaling.

The temperature gradient alters the energy exchanges. With the semi-local scaling, the energy exchanges have a lower spectral magnitude at the cold side than at the hot side, but handle a larger range of scales. Besides, the peaks of each energy exchanges are located at smaller wavenumbers and closer to the wall at the cold side than at the hot side. This is shown in table 2, which reports the spatial and spectral position and the amplitude of the peaks of each energy exchange. In addition, the spectra are provided in the set of figures 12 to 15 . The spectra of the viscous transfer and the convection, the two most significant parts of the conservative energy transfer, are given in figures 16 and 17 . With the semi-local scaling, the general behaviour of the spectral energy exchanges are similar at $R e_{\tau}=180$ and $R e_{\tau}=395$. As in the spatial domain, we consider that the spectral energy exchanges in the anisothermal configuration are in a large part due to the combination of the variations of the local fluid properties and a varying low Reynolds number effect. These two effects also influence the scales handled by the energy exchanges. A large part of the wavenumber asymmetry related to the variations of the local fluid properties is taken into account by the semi-local scaling. In accordance with the spatial results, the 


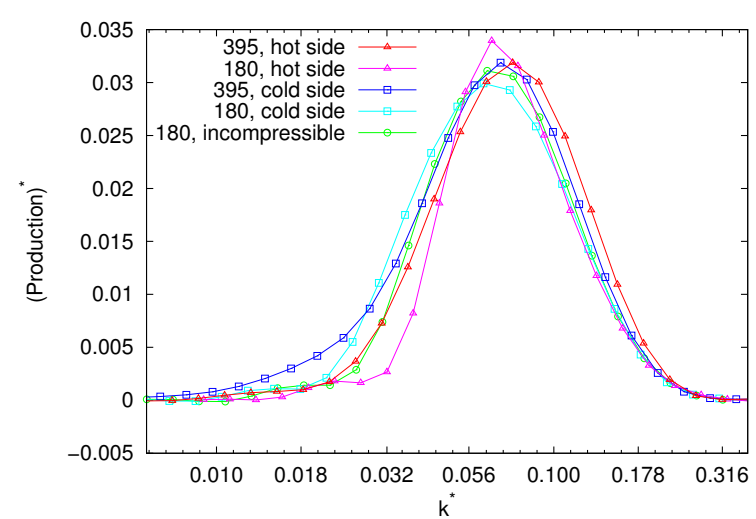

(a) Production $\check{\mathcal{P}}$.

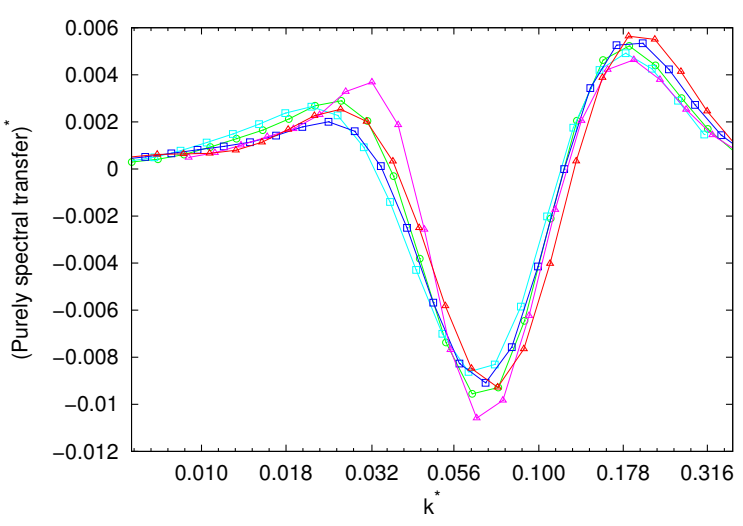

(c) Purely spectral transfer $\bar{\Xi}$.

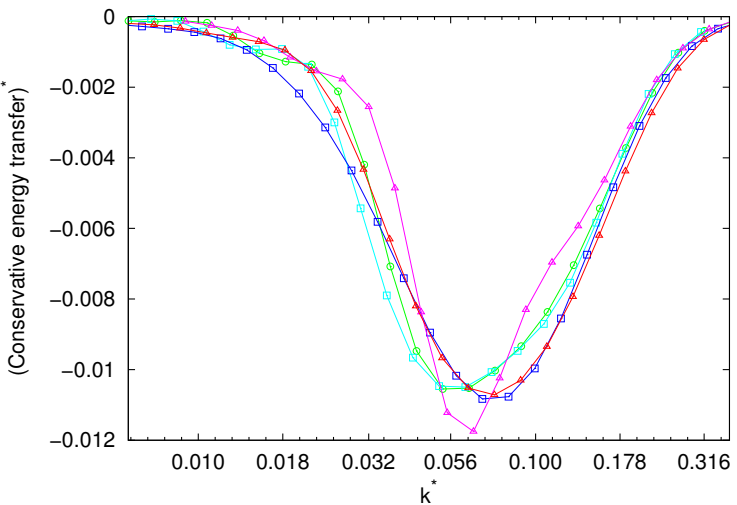

(b) Conservative energy transfer $\bar{\varphi}$.

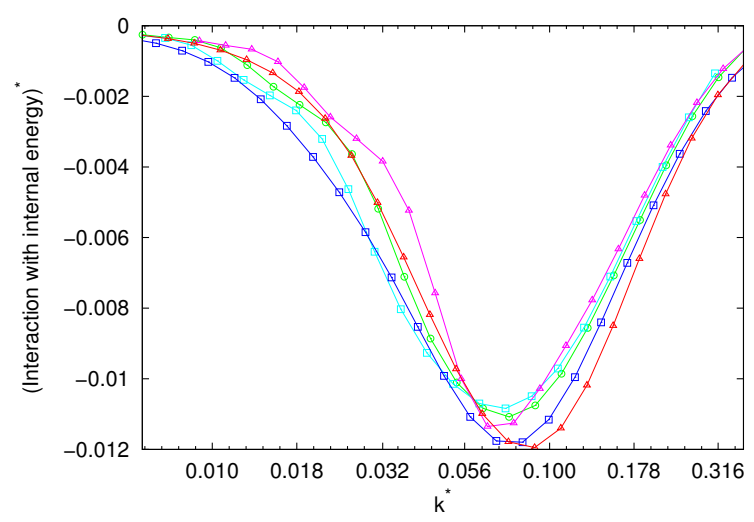

(d) Interaction with internal energy $\bar{\varepsilon}$.

Figure 9 - Profiles of the terms of the budget of the half-trace of the velocity covariance tensor with the semi-local scaling at $R e_{\tau}=180$ and $R e_{\tau}=395$ as a function of wavenumber at the location of the spatial peak of production $\left(y^{*} \approx 12\right)$.

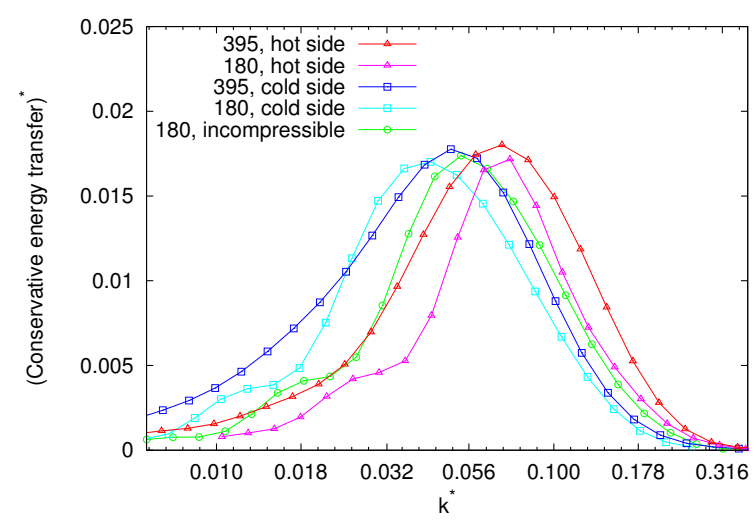

(a) Conservative energy transfer $\bar{\varphi}$.

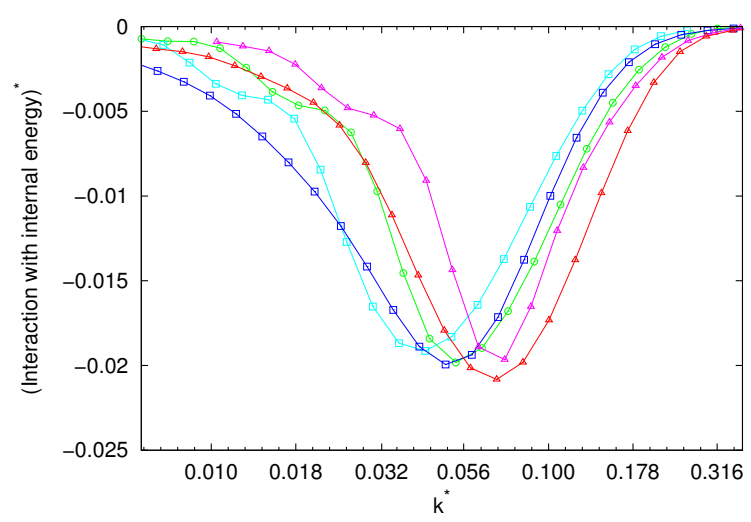

(b) Interaction with internal energy $\bar{\varepsilon}$.

Figure 10 - Profiles of the terms of the budget of the half-trace of the velocity covariance tensor with the semi-local scaling at $R e_{\tau}=180$ and $R e_{\tau}=395$ as a function of wavenumber very near to the wall (at $R e_{\tau}=180, y^{*}=0.25$ for the constant-property profile, $y^{*}=0.38$ at the cold side and $y^{*}=0.15$ at the hot side; at $R e_{\tau}=395, y^{*}=0.73$ at the cold side and $y^{*}=0.30$ at the hot side). The production and the purely spectral transfer are negligible. 


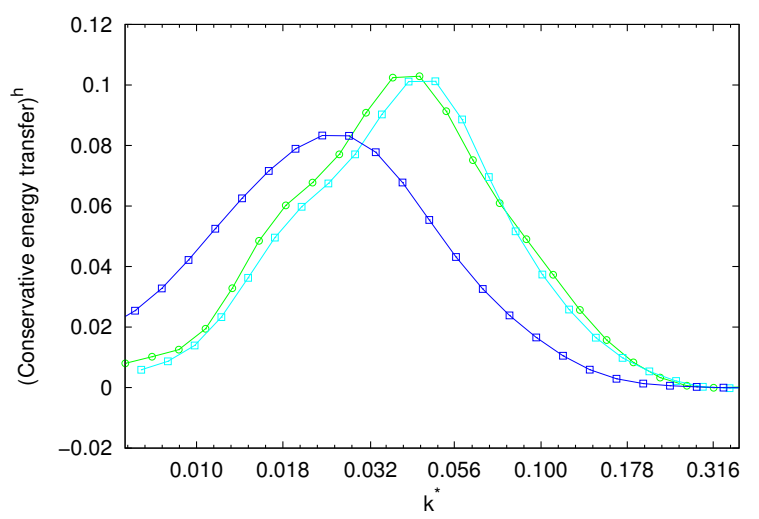

(a) Conservative energy transfer $\bar{\varphi}$.

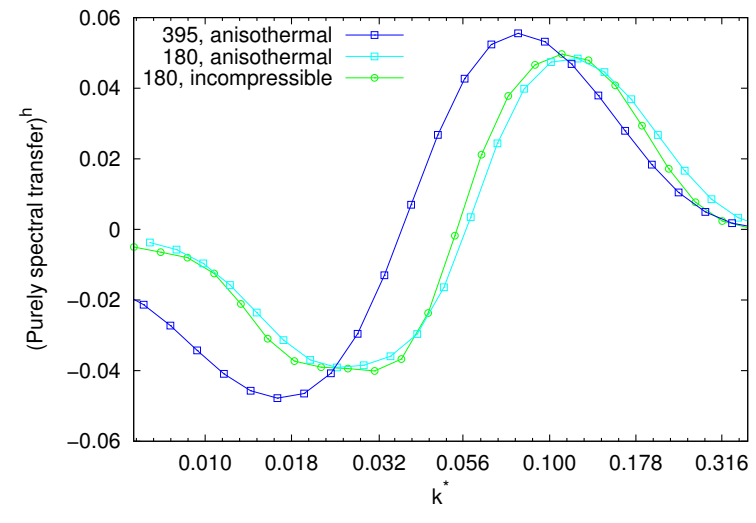

(b) Purely spectral transfer $\bar{\Xi}$.

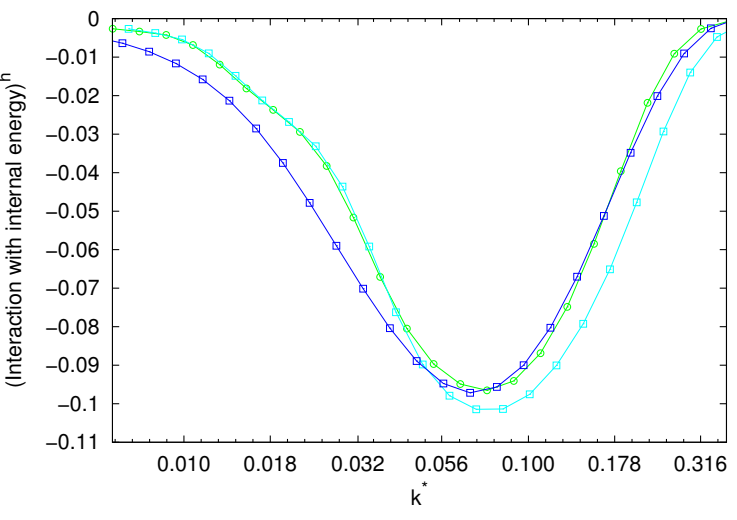

(c) Interaction with internal energy $\bar{\varepsilon}$.

Figure 11 - Profiles of the terms of the budget of the half-trace of the velocity covariance tensor scaled by $U_{\tau y}^{* 3} / h$ at $R e_{\tau}=180$ and $R e_{\tau}=395$ as a function of wavenumber at the centre of the channel. The production is negligible. In the anisothermal case, the hot and cold profiles are equal.

spectral extrema are closer to the wall at higher mean friction Reynolds number. This also applies to the interscale transport (figure 14), which has no contribution in the spatial domain. The spectra also suggest that the range of scales handled by the energy exchanges increases with the mean friction Reynolds number, mainly towards large but also towards small scales.

Besides these general effects relevant to all energy exchanges, the production peak (figure 12) is shifted towards small scales at $R e_{\tau}=395$ compared to $R e_{\tau}=180$ at the hot and cold sides. Its amplitude is increased at the cold side and decreased at the hot side, reducing the asymmetry between the hot and cold sides in terms of spectral magnitude. The negative peak of the conservative energy transfer (figure 13) follows the same pattern, thereby staying centred on the peak of production. The positive peak behaves like the extremum of the interaction with internal energy, described later. The negative extremum of the interscale transport (figure 14) is shifted towards small scales at the higher mean friction Reynolds number while the maximum at large scales is shifted towards large scales at the hot side and towards small scales at the cold side, reducing the asymmetry between the hot and cold sides. The negative area and the positive area at large scales have a smaller amplitude at $R e_{\tau}=395$ than at $R e_{\tau}=180$ whereas the positive area at small scales has a larger amplitude. The direct interscale transport towards small scales is 


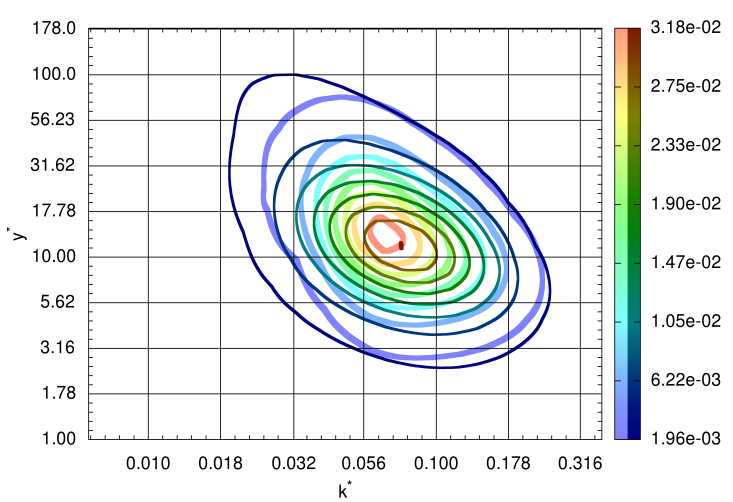

(a) Hot side, semi-local scaling.

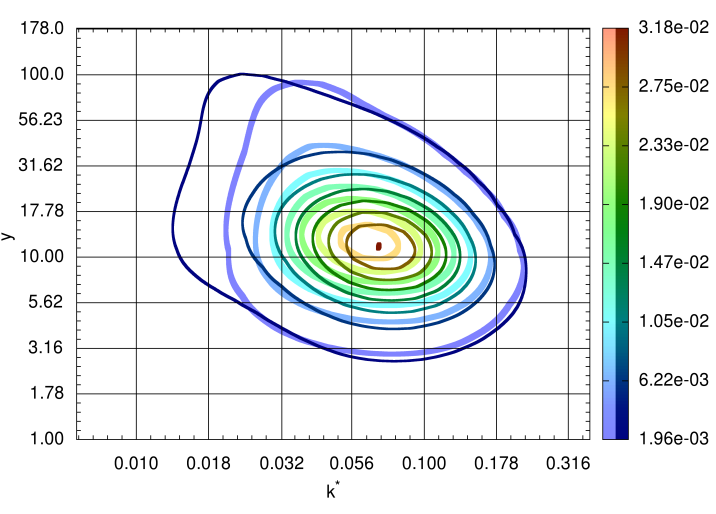

(b) Cold side, semi-local scaling.

Figure 12 - Spectra of the production $\overline{\mathcal{P}}$ with semi-local scaling at $R e_{\tau}=180$ (thick lines, light colour) and $R e_{\tau}=395$ (thin line, dark colour).

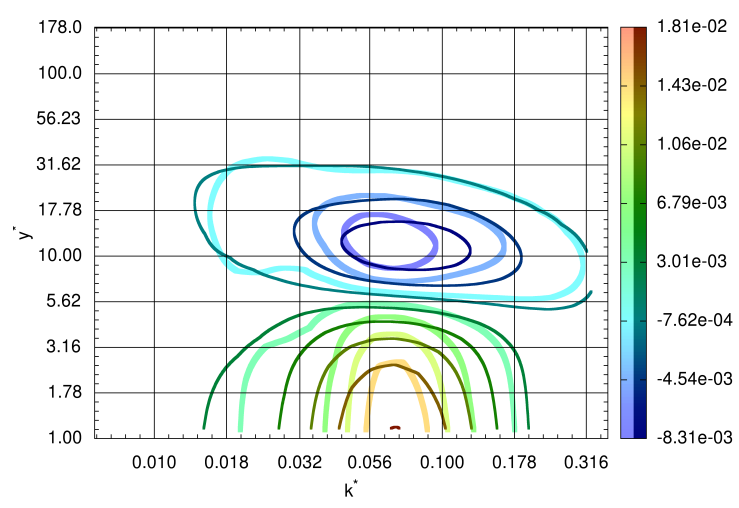

(a) Hot side, semi-local scaling.

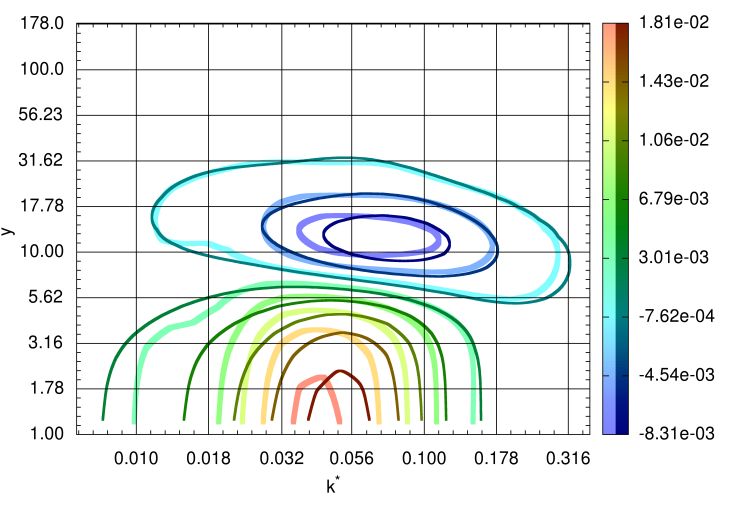

(b) Cold side, semi-local scaling.

Figure 13 - Spectra of the conservative energy transfer $\bar{\varphi}$ with semi-local scaling at $R e_{\tau}=$ 180 (thick lines, light colour) and $R e_{\tau}=395$ (thin line, dark colour).

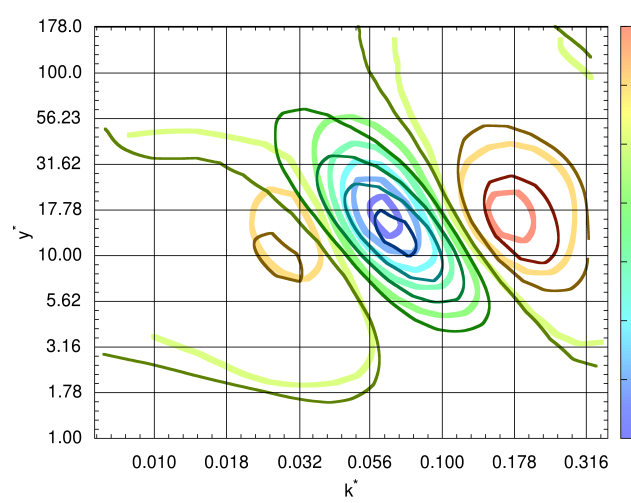

(a) Hot side, semi-local scaling.

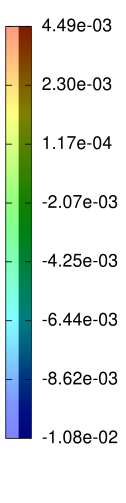

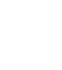

Figure 14 - Spectra of the purely spectral transfer $\Xi$ with semi-local scaling at $R e_{\tau}=180$ (thick lines, light colour) and $R e_{\tau}=395$ (thin line, dark colour). 


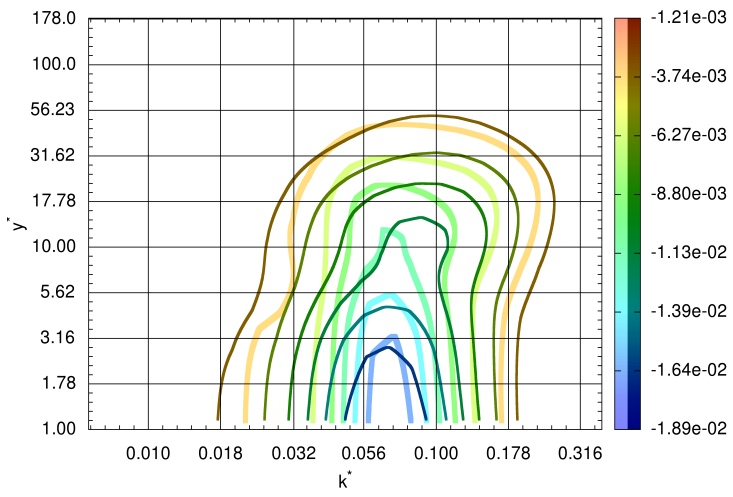

(a) Hot side, semi-local scaling.

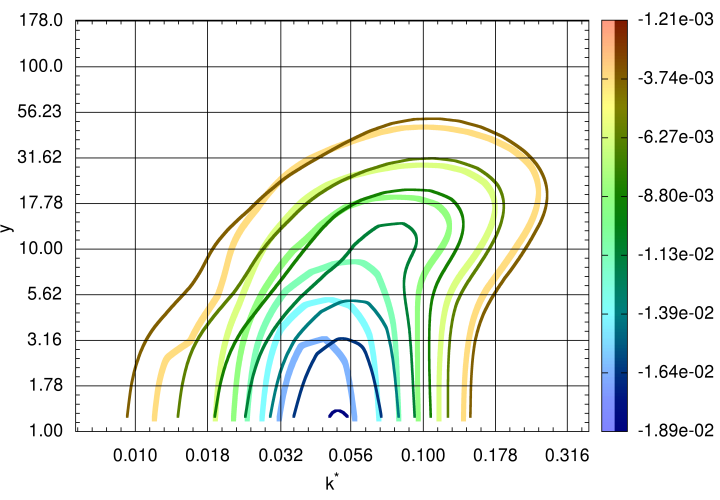

(b) Cold side, semi-local scaling.

Figure 15 - Spectra of the interaction with internal energy $\bar{\varepsilon}$ with semi-local scaling at $R e_{\tau}$ $=180$ (thick lines, light colour) and $R e_{\tau}=395$ (thin line, dark colour).

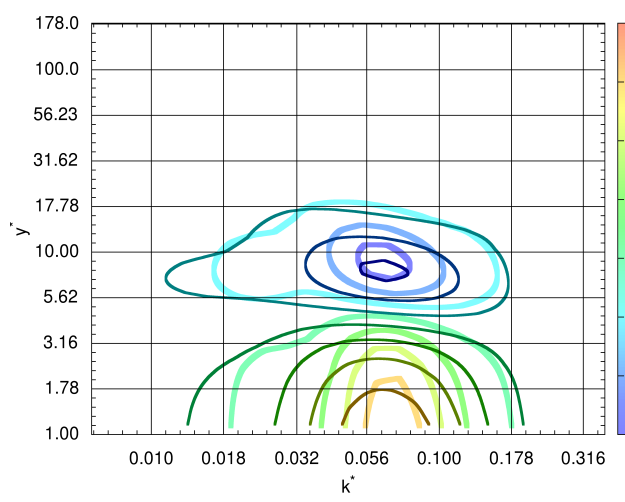

(a) Hot side, semi-local scaling.
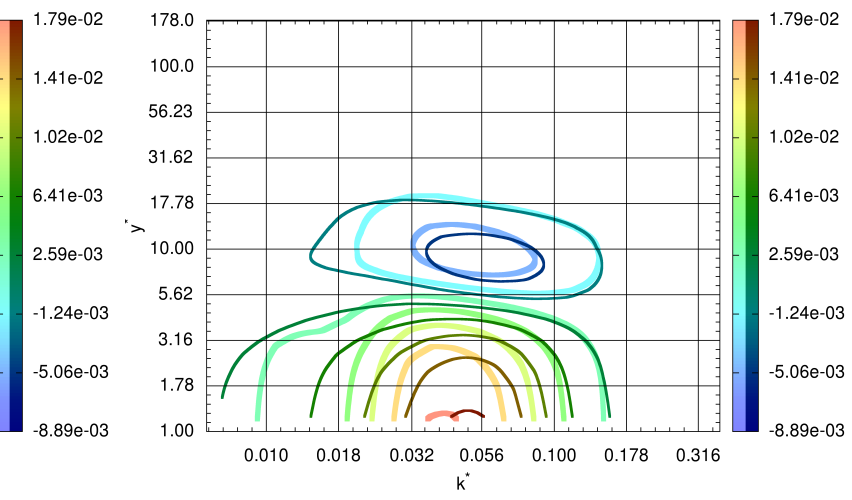

(b) Cold side, semi-local scaling.

Figure 16 - Spectra of the viscous transfer (part of the conservative energy transfer) $\bar{\varphi}^{\nu}$ with semi-local scaling at $R e_{\tau}=180$ (thick lines, light colour) and $R e_{\tau}=395$ (thin line, dark colour).

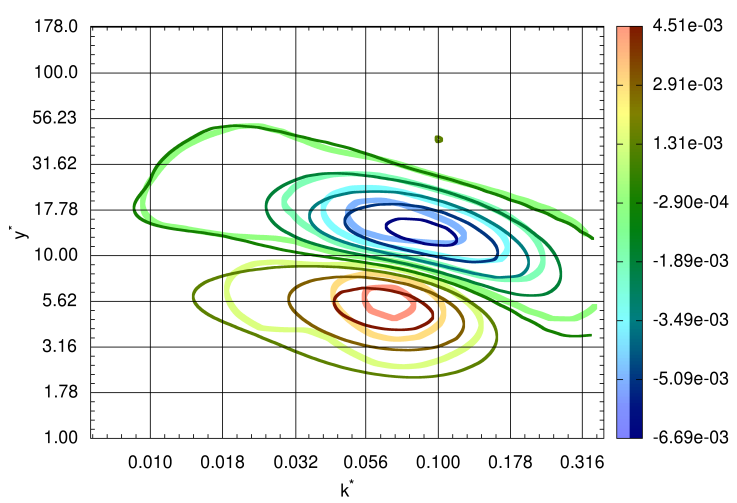

(a) Hot side, semi-local scaling.

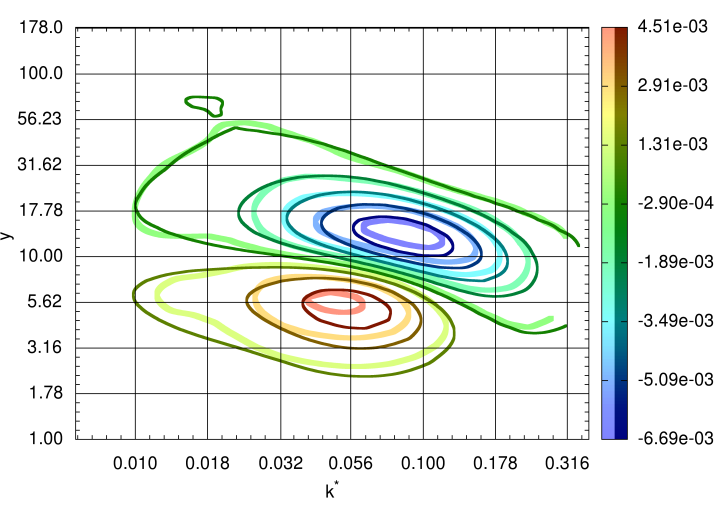

(b) Cold side, semi-local scaling.

Figure 17 - Spectra of the convection (part of the conservative energy transfer) $\overline{\breve{\varphi}^{c}}$ with semi-local scaling at $R e_{\tau}=180$ (thick lines, light colour) and $R e_{\tau}=395$ (thin line, dark colour). 


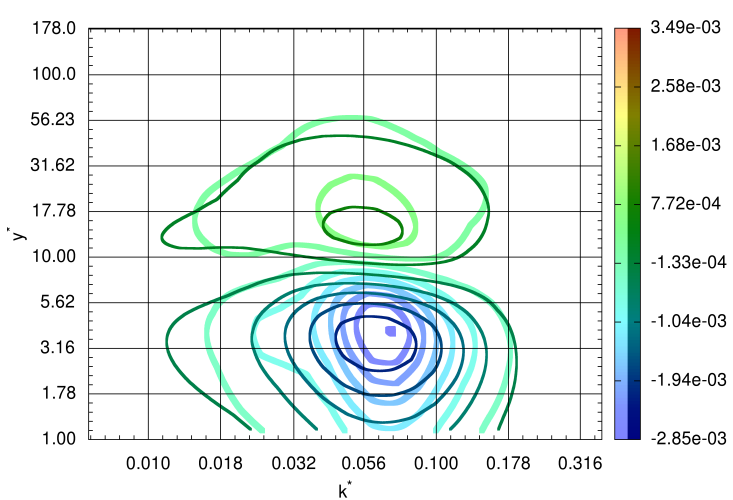

(a) Hot side, semi-local scaling.

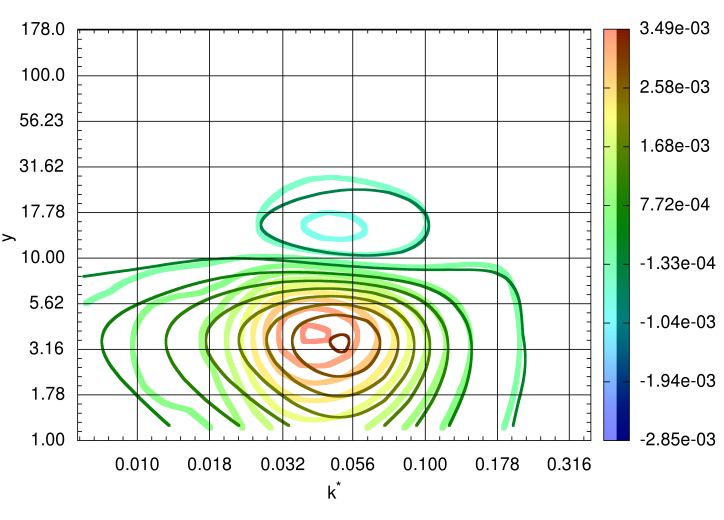

(b) Cold side, semi-local scaling.

Figure 18 - Spectra of the thermal conservative energy transfer $\bar{\varphi}_{\Gamma}$ with semi-local scaling at $R e_{\tau}=180$ (thick lines, light colour) and $R e_{\tau}=395$ (thin line, dark colour).

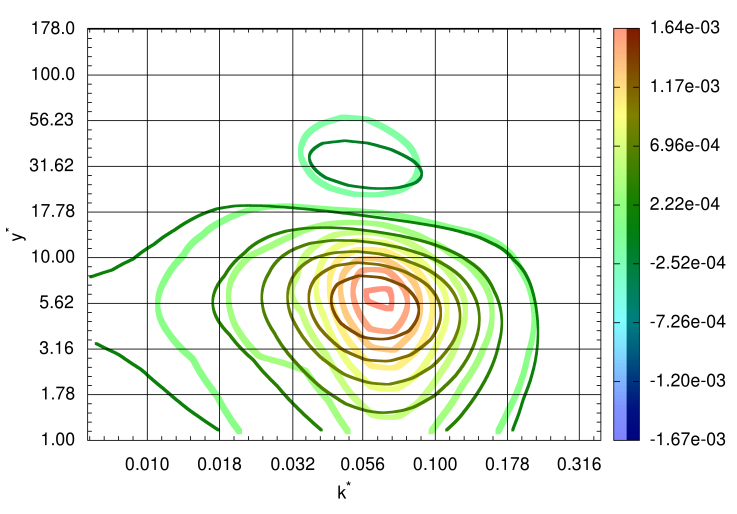

(a) Hot side, semi-local scaling.

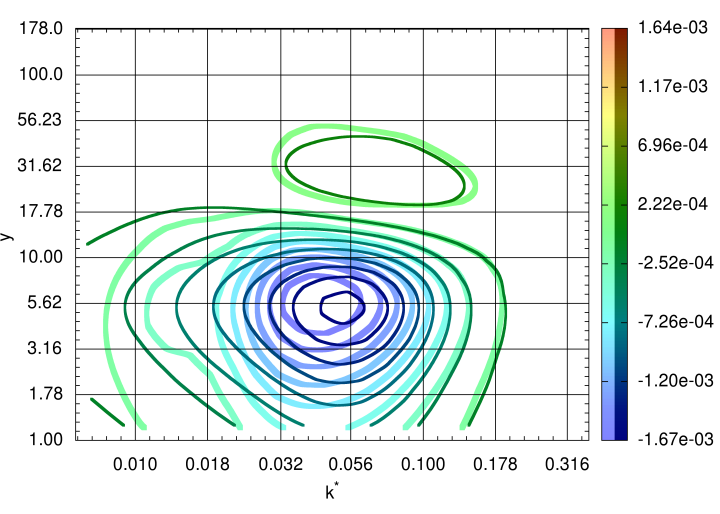

(b) Cold side, semi-local scaling.

Figure 19 - Spectra of the interaction with variable density kinetic energy $\bar{\zeta}$ with semi-local scaling at $R e_{\tau}=180$ (thick lines, light colour) and $R e_{\tau}=395$ (thin line, dark colour).

therefore strengthened compared to the transport towards large scales. The extremum of the interaction with internal energy (figure 15) has a larger amplitude at the higher mean friction Reynolds number. It is also shifted towards large scales at the hot side and towards small scales at the cold side, reducing the wavenumber asymmetry between the hot and cold side. This suggests that the wavenumber asymmetry between the hot and cold sides at $R e_{\tau}=180$ results in part from a low Reynolds number effect, that is from the semi-local friction Reynolds number asymmetry between the hot and cold side. The same remark can be made regarding the amplitude asymmetry of the production peak and the wavenumber asymmetry of the positive area at large scales of the interscale transport, although carefulness is required. A more precise knowledge of the different effects of the friction Reynolds number on the spectra in the constant-property isothermal case would be required for a more accurate separation of the different effects of the temperature gradient. 


\begin{tabular}{|c|c|c|c|c|c|c|c|}
\hline & \multirow[b]{2}{*}{ Side } & \multicolumn{3}{|c|}{$R e_{\tau}=180$} & \multirow[b]{2}{*}{$k^{*}$} & \multicolumn{2}{|c|}{$R e_{\tau}=395$} \\
\hline & & $k^{*}$ & $y^{*}$ & Amplitude & & $y^{*}$ & Amplitude \\
\hline \multirow{2}{*}{ Production } & $\mathrm{C}$ & 0.062 & 12.4 & $3.00 \times 10^{-2}$ & 0.069 & 11.2 & $3.19 \times 10^{-2}$ \\
\hline & $\mathrm{H}$ & 0.065 & 13.5 & $3.42 \times 10^{-2}$ & 0.076 & 11.6 & $3.19 \times 10^{-2}$ \\
\hline \multirow{4}{*}{ Conservative energy transfer } & $\mathrm{C}$ & 0.043 & 0.64 & $1.92 \times 10^{-2}$ & 0.050 & 1.21 & $1.97 \times 10^{-2}$ \\
\hline & $\mathrm{H}$ & 0.074 & 0.26 & $1.93 \times 10^{-2}$ & 0.070 & 0.50 & $2.00 \times 10^{-2}$ \\
\hline & $\mathrm{C}$ & 0.052 & 12.4 & $-1.05 \times 10^{-2}$ & 0.070 & 11.7 & $-1.08 \times 10^{-2}$ \\
\hline & $\mathrm{H}$ & 0.066 & 11.9 & $-1.18 \times 10^{-2}$ & 0.076 & 11.2 & $-1.07 \times 10^{-2}$ \\
\hline \multirow{6}{*}{ Purely spectral transfer } & $\mathrm{C}$ & 0.021 & 12.4 & $2.65 \times 10^{-3}$ & 0.023 & 9.76 & $2.25 \times 10^{-3}$ \\
\hline & $\mathrm{H}$ & 0.032 & 13.5 & $3.73 \times 10^{-3}$ & 0.026 & 10.4 & $2.59 \times 10^{-3}$ \\
\hline & $\mathrm{C}$ & 0.063 & 14.0 & $-9.13 \times 10^{-3}$ & 0.070 & 11.7 & $-9.08 \times 10^{-3}$ \\
\hline & $\mathrm{H}$ & 0.064 & 16.5 & $-1.21 \times 10^{-2}$ & 0.075 & 12.4 & $-9.33 \times 10^{-3}$ \\
\hline & $\mathrm{C}$ & 0.186 & 14.6 & $5.29 \times 10^{-3}$ & 0.174 & 14.8 & $5.77 \times 10^{-3}$ \\
\hline & $\mathrm{H}$ & 0.187 & 15.8 & $5.07 \times 10^{-3}$ & 0.181 & 15.3 & $6.24 \times 10^{-3}$ \\
\hline \multirow{2}{*}{ Interaction with internal energy } & $\mathrm{C}$ & 0.043 & 0.38 & $-1.91 \times 10^{-2}$ & 0.049 & 0.73 & $-1.99 \times 10^{-2}$ \\
\hline & $\mathrm{H}$ & 0.074 & 0.15 & $-1.97 \times 10^{-2}$ & 0.070 & 0.30 & $-2.08 \times 10^{-2}$ \\
\hline \multirow{4}{*}{ Viscous transfer } & $\mathrm{C}$ & 0.043 & 0.64 & $1.88 \times 10^{-2}$ & 0.050 & 1.21 & $1.84 \times 10^{-2}$ \\
\hline & $\mathrm{H}$ & 0.074 & 0.26 & $1.90 \times 10^{-2}$ & 0.070 & 0.50 & $1.91 \times 10^{-2}$ \\
\hline & $\mathrm{C}$ & 0.050 & 9.56 & $-7.81 \times 10^{-3}$ & 0.056 & 8.80 & $-7.50 \times 10^{-3}$ \\
\hline & $\mathrm{H}$ & 0.067 & 8.71 & $-1.06 \times 10^{-2}$ & 0.065 & 7.95 & $-9.45 \times 10^{-3}$ \\
\hline \multirow{4}{*}{ Convection } & $\mathrm{C}$ & 0.047 & 5.58 & $4.75 \times 10^{-3}$ & 0.054 & 5.48 & $5.51 \times 10^{-3}$ \\
\hline & $\mathrm{H}$ & 0.070 & 5.62 & $5.20 \times 10^{-3}$ & 0.067 & 5.15 & $5.62 \times 10^{-3}$ \\
\hline & $\mathrm{C}$ & 0.090 & 13.5 & $-7.29 \times 10^{-3}$ & 0.084 & 13.2 & $-8.09 \times 10^{-3}$ \\
\hline & $\mathrm{H}$ & 0.064 & 15.8 & $-6.52 \times 10^{-3}$ & 0.089 & 13.6 & $-7.30 \times 10^{-3}$ \\
\hline \multirow{4}{*}{$\begin{array}{l}\text { Thermal conservative energy } \\
\text { transfer }\end{array}$} & $\mathrm{C}$ & 0.038 & 3.87 & $3.77 \times 10^{-3}$ & 0.052 & 3.59 & $3.33 \times 10^{-3}$ \\
\hline & $\mathrm{H}$ & 0.071 & 4.03 & $-3.08 \times 10^{-3}$ & 0.057 & 3.51 & $-2.49 \times 10^{-3}$ \\
\hline & $\mathrm{C}$ & 0.045 & 15.1 & $-9.19 \times 10^{-4}$ & 0.059 & 13.8 & $-6.94 \times 10^{-4}$ \\
\hline & $\mathrm{H}$ & 0.053 & 16.5 & $9.90 \times 10^{-4}$ & 0.052 & 14.5 & $6.51 \times 10^{-4}$ \\
\hline \multirow{4}{*}{$\begin{array}{l}\text { Interaction with variable density } \\
\text { kinetic energy }\end{array}$} & $\mathrm{C}$ & 0.040 & 5.94 & $-2.04 \times 10^{-3}$ & 0.054 & 5.48 & $-1.88 \times 10^{-3}$ \\
\hline & $\mathrm{H}$ & 0.069 & 5.92 & $1.80 \times 10^{-3}$ & 0.066 & 5.44 & $1.51 \times 10^{-3}$ \\
\hline & $\mathrm{C}$ & 0.057 & 27.5 & $2.09 \times 10^{-4}$ & 0.075 & 27.2 & $1.73 \times 10^{-4}$ \\
\hline & $\mathrm{H}$ & 0.050 & 31.6 & $-2.56 \times 10^{-4}$ & 0.059 & 30.3 & $-1.66 \times 10^{-4}$ \\
\hline \multirow{4}{*}{$\begin{array}{l}\text { Thermal interaction with internal } \\
\text { energy }\end{array}$} & $\mathrm{C}$ & 0.039 & 4.53 & $-1.55 \times 10^{-3}$ & 0.052 & 4.07 & $-1.37 \times 10^{-3}$ \\
\hline & $\mathrm{H}$ & 0.070 & 4.79 & $1.34 \times 10^{-3}$ & 0.056 & 4.30 & $1.05 \times 10^{-3}$ \\
\hline & $\mathrm{C}$ & 0.160 & 19.6 & $3.53 \times 10^{-4}$ & 0.150 & 19.8 & $3.15 \times 10^{-4}$ \\
\hline & $\mathrm{H}$ & 0.150 & 24.8 & $-2.39 \times 10^{-4}$ & 0.147 & 22.7 & $-2.22 \times 10^{-4}$ \\
\hline
\end{tabular}

Table 2 - Wavenumber, wall-normal coordinate and amplitude of the local spectral extrema of each term of the equation of the half-trace of the velocity covariance tensor with the semi-local scaling at the hot $(\mathrm{H})$ and cold $(\mathrm{C})$ sides at $R e_{\tau}=180$ and $R e_{\tau}=395$ in the anisothermal configuration. 


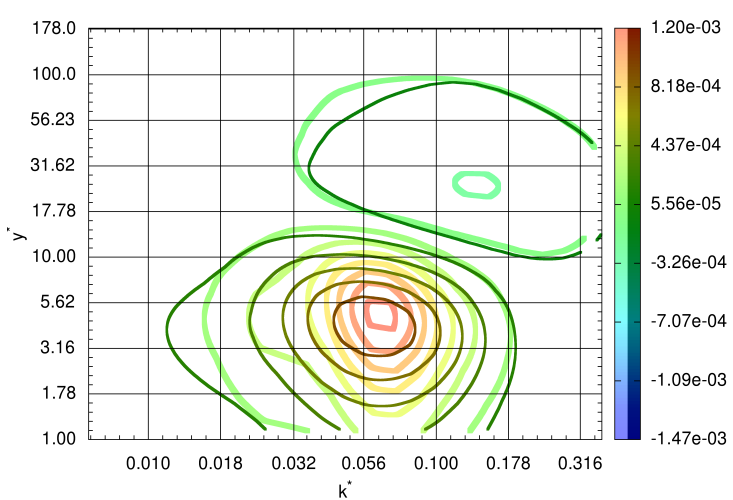

(a) Hot side, semi-local scaling.

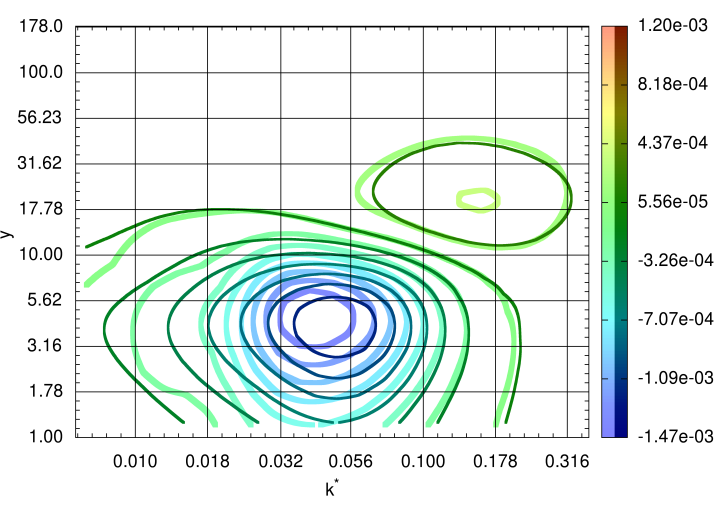

(b) Cold side, semi-local scaling.

Figure 20 - Spectra of the thermal interaction with internal energy $\bar{\varepsilon}_{\Gamma}$ with semi-local scaling at $R e_{\tau}=180$ (thick lines, light colour) and $R e_{\tau}=395$ (thin line, dark colour).

\subsubsection{Spectra of the thermal terms in the anisothermal configuration}

The spectra of the three most significant thermal terms include the spectra of the interaction with variable density kinetic energy $\bar{\zeta}$, a thermal energy exchange, and the thermal conservative energy transfer $\bar{\varphi}_{\Gamma}$ and the thermal interaction with internal energy $\bar{\varepsilon}_{\Gamma}$, the thermal parts of energy exchanges. They are given with the semi-local scaling at $R e_{\tau}=180$ and $R e_{\tau}=395$ in the set of figures 18 to 20 . The effect of the mean friction Reynolds number on the spatial profiles of the thermal terms was found to be small. In the spectral domain, more differences are identified. For the three thermal terms (figures 18 to 20), the amplitude of the extremum close to the wall is decreased at the hot and cold sides at $R e_{\tau}=395$ compared to $R e_{\tau}=180$, by around $20 \%$ at the hot side and by around $10 \%$ at the cold side. This is rather unexpected since the amplitude of the spatial profiles is largely unaffected by the mean friction Reynolds number. The lower spectral magnitude are counterbalanced by the larger range of scales handled. In addition to a spatial shift towards the wall affecting all terms, the extremum close to the wall is shifted towards large scales at the hot side and towards small scales at the cold side. This reduces the wavenumber asymmetry between the hot and cold sides and suggests that this asymmetry is tied to a low Reynolds number effect. The mean friction Reynolds number seems to modify the spectral behaviour of the thermal terms but does not alter significantly their spatial profiles within the Reynolds number range of this study.

\subsubsection{Kinetic energy spectrum}

The energy exchanges drive the spectrum of the half-trace of the velocity covariance tensor, which is also asymmetrised between the hot and cold sides. Figure 21 gives the streamwise spectra in the anisothermal channels at $R e_{\tau}=180$ and $R e_{\tau}=395$ at the location of the spatial peak of production. To compare the slope of the different spectra at the hot and cold sides, the spectra are normalised to one at the smallest wavenumber common to all simulations. The slope of the spectrum is increased at the hot side and decreased at the cold side. The effect is consistent with an effect of the semi-local friction Reynolds number as shown by the reference data of Moser et al. [46]. We are not able to distinguish an effect of the variations of the fluid properties on the slope of the spectrum. 


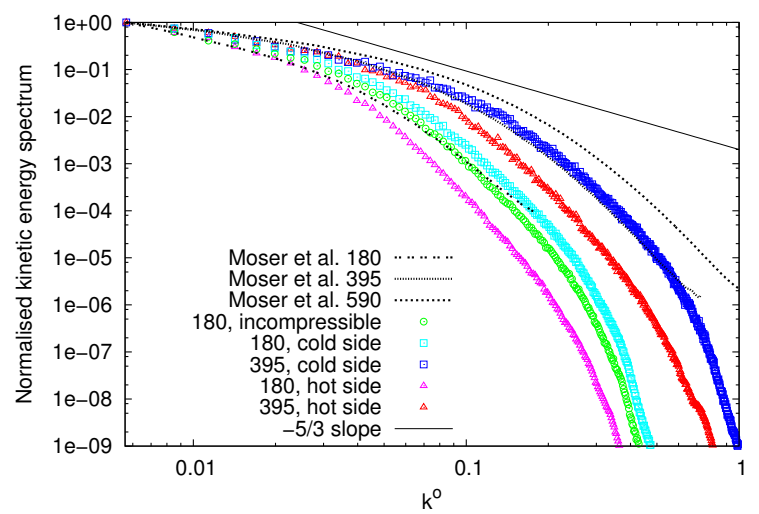

Figure 21 - Normalised streamwise spectrum of the half-trace of the velocity covariance tensor at $R e_{\tau}=180$ and $R e_{\tau}=395$ at the location of the spatial peak of production $\left(y^{*} \approx\right.$ $12)$.

\section{Conclusion}

The effect of the Reynolds number on the energy exchanges associated with the halftrace of the velocity covariance tensor is investigated in the spatial and spectral domains in a strongly anisothermal low Mach fully developed turbulent channel flow. The study is based on the direct numerical simulations of the channel at the mean friction Reynolds number of $R e_{\tau}=180$ and $R e_{\tau}=395$. The temperature gradient creates an asymmetry between the energy exchanges at the hot and cold side. The asymmetry can be attributed to the combined effect of the variations of the local fluid properties and a varying low Reynolds number effect, based on the semi-local friction Reynolds number which varies across the channel. The effect of the variations of the local fluid properties is to some extent taken into account by the semi-local scaling, defined using the wall shear-stress and the mean local fluid properties. The low Reynolds number effects, not taken into account by the semi-local scaling, are investigated using reference data in the constant-property isothermal channel. The effects of the semi-local friction Reynolds number variations are smaller at higher mean friction Reynolds number, reducing the asymmetry between the hot and cold sides. With the semi-local scaling, the energy exchanges occur closer to the wall at $R e_{\tau}=395$ than at $R e_{\tau}=180$, handle a larger range of scales and have a larger spatial amplitude. The energy exchanges are decomposed to isolate the terms specific to flows with variable fluid properties, called thermal terms. The mean friction Reynolds number modifies the spectral behaviour of the thermal terms but does not affect significantly their spatial profile.

\section{Acknowledgments}

The authors gratefully acknowledge the CEA for the development of the TRUST platform and TrioCFD. This work was granted access to the HPC resources of CINES under the allocations 2017-A0022A05099 and 2018-A0042A05099 made by GENCI. 


\section{References}

[1] H. Abe, H. Kawamura, and Y. Matsuo. Direct numerical simulation of a fully developed turbulent channel flow with respect to the Reynolds number dependence. Journal of Fluids Engineering, 123(2):382-393, 2001.

[2] J. R. Andrade, R. S. Martins, G. Mompean, L. Thais, and T. B. Gatski. Analyzing the spectral energy cascade in turbulent channel flow. Physics of Fluids, 30(6):065110, 2018.

[3] F. Aulery, A. Toutant, F. Bataille, and Y. Zhou. Energy transfer process of anisothermal wall-bounded flows. Physics Letters A, 379(24):1520-1526, 2015.

[4] F. Aulery, D. Dupuy, A. Toutant, F. Bataille, and Y. Zhou. Spectral analysis of turbulence in anisothermal channel flows. Computers \& Fluids, 151:115-131, 2017.

[5] B. Aupoix. Introduction to turbulence modelling for compressible flows. VKI lecture series, 4:H1-H64, 2000.

[6] K. Blackman, L. Perret, I. Calmet, and C. Rivet. Turbulent kinetic energy budget in the boundary layer developing over an urban-like rough wall using piv. Physics of Fluids, 29(8): $085113,2017$.

[7] I. A. Bolotnov, R. T. Lahey, D. A. Drew, K. E. Jansen, and A. A. Oberai. Spectral analysis of turbulence based on the DNS of a channel flow. Computers \& Fluids, 39(4):640-655, 2010.

[8] P. Bradshaw. Compressible turbulent shear layers. Annual Review of Fluid Mechanics, 9(1): $33-52,1977$.

[9] C. Calvin, O. Cueto, and P. Emonot. An object-oriented approach to the design of fluid mechanics software. ESAIM: Mathematical Modelling and Numerical Analysis, 36(05):907921, 2002.

[10] M. Campolo, M. Andreoli, and A. Soldati. Computing flow, combustion, heat transfer and thrust in a micro-rocket via hierarchical problem decomposition. Microfluidics and nanofluidics, 7(1):57-73, 2009.

[11] P. Chassaing, R. A. Antonia, F. Anselmet, L. Joly, and S. Sarkar. Variable density fluid turbulence. Springer Science \& Business Media, 2013.

[12] A. W. Cook and Y. Zhou. Energy transfer in Rayleigh-Taylor instability. Physical Review E, 66:026312, 2002.

[13] J. Cousteix and B. Aupoix. Turbulence models for compressible flows. In Special Course Three-Dimensional Supersonic and Hypersonic Flows Including Separation. AGARD/FDPVKI Special course, 1989.

[14] X. Daguenet-Frick, J.-M. Foucaut, S. Coudert, A. Toutant, and G. Olalde. Experimental analysis of the turbulent flow behavior of a textured surface proposed for asymmetric heat exchangers. Flow, Turbulence and Combustion, 89(1):149-169, 2012.

[15] X. Daguenet-Frick, A. Toutant, F. Bataille, and G. Olalde. Numerical investigation of a ceramic high-temperature pressurized-air solar receiver. Solar Energy, 90(0):164-178, 2013. ISSN 0038-092X.

[16] J. C. Del Álamo and J. Jiménez. Spectra of the very large anisotropic scales in turbulent channels. Phyics of Fluids, 15(6):L41-L44, 2003.

[17] J. C. Del Álamo, J. Jiménez, P. Zandonade, and R. D. Moser. Scaling of the energy spectra of turbulent channels. Journal of Fluid Mechanics, 500:135-144, 2004.

[18] J. A. Domaradzki, W. Liu, C. Hartel, and L. Kleiser. Energy transfer in numerically simulated wall-bounded turbulent flows. Phyics of Fluids, 6(4):1583-1599, 1994.

[19] D. Dupuy, A. Toutant, and F. Bataille. Equations of energy exchanges in variable density turbulent flows. Physics Letters A, 382(5):327-333, 2018.

[20] D. Dupuy, A. Toutant, and F. Bataille. Turbulence kinetic energy exchanges in flows with highly variable fluid properties. Journal of Fluid Mechanics, 834:5-54, 2018.

[21] O. Flores and J. Jiménez. Hierarchy of minimal flow units in the logarithmic layer. Physics of Fluids, 22(7):071704, 2010.

[22] S. Gamard and W. K. George. Reynolds number dependence of energy spectra in the overlap region of isotropic turbulence. Flow, turbulence and combustion, 63(1-4):443-477, 2000.

[23] T. B. Gatski and J. P. Bonnet. Compressibility, turbulence and high speed flow. Academic Press, 2013. 
[24] S. Hoyas and J. Jiménez. Scaling of the velocity fluctuations in turbulent channels up to $\operatorname{Re}_{\tau}=2003$. Phyics of Fluids, 18(1):011702, 2006.

[25] S. Hoyas and J. Jiménez. Reynolds number effects on the reynolds-stress budgets in turbulent channels. Physics of Fluids, 20(10):101511, 2008.

[26] P. G. Huang, G. N. Coleman, and P. Bradshaw. Compressible turbulent channel flows: DNS results and modelling. Journal of Fluid Mechanics, 305:185-218, 1995.

[27] N. Hutchins and I. Marusic. Large-scale influences in near-wall turbulence. Philosophical Transactions of the Royal Society of London A: Mathematical, Physical and Engineering Sciences, 365(1852):647-664, 2007.

[28] J. Jiménez. The physics of wall turbulence. Physica A: Statistical Mechanics and its Applications, 263(1-4):252-262, 1999.

[29] J. Jiménez. Near-wall turbulence. Physics of Fluids, 25(10):101302, 2013.

[30] J. Jiménez and A. Pinelli. The autonomous cycle of near-wall turbulence. Journal of Fluid Mechanics, 389:335-359, 1999.

[31] Javier Jiménez and Robert D Moser. What are we learning from simulating wall turbulence? Philosophical Transactions of the Royal Society of London A: Mathematical, Physical and Engineering Sciences, 365(1852):715-732, 2007.

[32] S. Kida and S. A. Orszag. Energy and spectral dynamics in decaying compressible turbulence. Journal of Scientific Computing, 7(1):1-34, 1992. ISSN 0885-7474.

[33] J. Kim, P. Moin, and R. Moser. Turbulence statistics in fully developed channel flow at low Reynolds number. Journal of Fluid Mechanics, 177:133-166, 1987.

[34] F. Laadhari. On the evolution of maximum turbulent kinetic energy production in a channel flow. Phyics of Fluids, 14(10):L65-L68, 2002.

[35] J. Lee, P. E. Gharagozloo, B. Kolade, J. K. Eaton, and K. E. Goodson. Nanofluid convection in microtubes. Journal of heat transfer, 132(9):092401, 2010.

[36] J. Lee, S. Y. Jung, H. J. Sung, and T. A. Zaki. Effect of wall heating on turbulent boundary layers with temperature-dependent viscosity. Journal of Fluid Mechanics, 726:196-225, 2013.

[37] M. Lee and R. D. Moser. Spectral analysis on reynolds stress transport equation in high firewall-bounded turbulence. In International Symposium on Turbulence and Shear Flow Phenomena (TSFP-9), Melbourne, pages 4A-3, 2006.

[38] M. Lee and R. D. Moser. Direct numerical simulation of turbulent channel flow up to $\operatorname{Re}_{\tau} \approx$ 5200. Journal of Fluid Mechanics, 774:395-415, 2015.

[39] S. K. Lele. Compressibility effects on turbulence. Annual Review of Fluid Mechanics, 26(1): 211-254, 1994.

[40] M. Lesieur. Turbulence in Fluids. Fluid Mechanics and Its Applications. Springer, 2008. ISBN 9781402064357.

[41] R. Mathis, N. Hutchins, and I. Marusic. Large-scale amplitude modulation of the small-scale structures in turbulent boundary layers. Journal of Fluid Mechanics, 628:311-337, 2009.

[42] A. Meister. Asymptotic single and multiple scale expansions in the low mach number limit. SIAM Journal on Applied Mathematics, 60(1):256-271, 1999.

[43] Y. Mizuno. Spectra of energy transport in turbulent channel flows for moderate reynolds numbers. Journal of Fluid Mechanics, 805:171-187, 2016.

[44] J.-P. Mollicone, F. Battista, P. Gualtieri, and C. M. Casciola. Turbulence dynamics in separated flows: the generalised kolmogorov equation for inhomogeneous anisotropic conditions. Journal of Fluid Mechanics, 841:1012-1039, 2018.

[45] Y. Morinishi, T. S. Lundhomas, O. V. Vasilyev, and P. Moin. Fully conservative higher order finite difference schemes for incompressible flow. J. Comput. Phys., 143(1):90-124, 1998.

[46] R. D. Moser, J. Kim, and N. N. Mansour. Direct numerical simulation of turbulent channel flow up to $\operatorname{Re}_{\tau}=590$. Phyics of Fluids, 11(4):943-945, 1999.

[47] H. Nemati, A. Patel, B. J. Boersma, and R. Pecnik. Mean statistics of a heated turbulent pipe flow at supercritical pressure. International Journal of Heat and Mass Transfer, 83:741-752, 2015.

[48] F. Nicoud. Conservative high-order finite-difference schemes for low-Mach number flows. $J$. Comput. Phys., 158(1):71-97, 2000.

[49] S. Paolucci. On the filtering of sound from the Navier-Stokes equations. Technical Report SAND82-8257, Sandia National Labs., Livermore, CA (USA), 1982. 
[50] A. Patel, B. J. Boersma, and R. Pecnik. Scalar statistics in variable property turbulent channel flows. Physical Review Fluids, 2(8):084604, 2017.

[51] R. Pecnik and A. Patel. Scaling and modelling of turbulence in variable property channel flows. Journal of Fluid Mechanics, 823, 2017.

[52] J. W. R. Peeters, R. Pecnik, M. Rohde, T. H. J. J. Van Der Hagen, and B. J. Boersma. Turbulence attenuation in simultaneously heated and cooled annular flows at supercritical pressure. Journal of Fluid Mechanics, 799:505-540, 2016.

[53] M. Sanchez, F. Aulery, A. Toutant, and F. Bataille. Large eddy simulation of thermal boundary layer spatial development in a turbulent channel flow. Journal of Fluids Engineering, 136 (6):060906, 2014.

[54] L. A. C. A. Schiavo, W. R. Wolf, and J. L. F. Azevedo. Turbulent kinetic energy budgets in wall bounded flows with pressure gradients and separation. Physics of Fluids, 29(11):115108, 2017.

[55] S. Serra, A. Toutant, and F. Bataille. Thermal large eddy simulation in a very simplified geometry of a solar receiver. Heat Transfer Engineering, 33(6):505-524, 2012.

[56] S. Serra, A. Toutant, F. Bataille, and Y. Zhou. High-temperature gradient effect on a turbulent channel flow using thermal large-eddy simulation in physical and spectral spaces. $J$. Turbulence, 13:N49, 2012.

[57] S. Serra, A. Toutant, F. Bataille, and Y. Zhou. Turbulent kinetic energy spectrum in very anisothermal flows. Physics Letters A, 376(45):3177-3184, 2012.

[58] M. K. Sharma, A. Kumar, M. K. Verma, and S. Chakraborty. Statistical features of rapidly rotating decaying turbulence: Enstrophy and energy spectra and coherent structures. Physics of Fluids, 30(4):045103, 2018.

[59] M. K. Sharma, M. K. Verma, and S. Chakraborty. On the energy spectrum of rapidly rotating forced turbulence. Physics of Fluids, 30(11):115102, 2018.

[60] A. J. Smits, B. J. McKeon, and I. Marusic. High-reynolds number wall turbulence. Annual Review of Fluid Mechanics, 43, 2011.

[61] W. Sutherland. The viscosity of gases and molecular force. The London, Edinburgh, and Dublin Philosophical Magazine and Journal of Science, 36(223):507-531, 1893.

[62] A. Toutant and F. Bataille. Turbulence statistics in a fully developed channel flow submitted to a high temperature gradient. International Journal of Thermal Sciences, 74:104-118, 2013.

[63] C. A. Z. Towery, A. Y. Poludnenko, J. Urzay, J. O'Brien, M. Ihme, and P. E. Hamlington. Spectral kinetic energy transfer in turbulent premixed reacting flows. Physical Review E, 93 (5):053115, 2016.

[64] A. W. Vreman and J. G. M. Kuerten. Comparison of direct numerical simulation databases of turbulent channel flow at $\mathrm{Re}_{\tau}=180$. Phyics of Fluids, 26(1):015102, 2014.

[65] J. H. Williamson. Low-storage Runge-Kutta schemes. J. Comput. Phys., 35(1):48-56, 1980.

[66] Y. Yang, W. H. Matthaeus, T. N. Parashar, C. C. Haggerty, V. Roytershteyn, W. Daughton, M. Wan, Y. Shi, and S. Chen. Energy transfer, pressure tensor, and heating of kinetic plasma. Physics of Plasmas, 24(7):072306, 2017.

[67] Y. Yang, W. H. Matthaeus, T. N. Parashar, P. Wu, M. Wan, Y. Shi, S. Chen, V. Roytershteyn, and W. Daughton. Energy transfer channels and turbulence cascade in vlasov-maxwell turbulence. Physical Review E, 95(6):061201, 2017.

[68] J. Y. Yoo. The turbulent flows of supercritical fluids with heat transfer. Annual review of fluid mechanics, 45:495-525, 2013.

[69] Y. Zhou. Renormalization group theory for fluid and plasma turbulence. Physics Reports, 488(1):1-49, 2010.

[70] Y. Zhou and S. Oughton. Nonlocality and the critical Reynolds numbers of the minimum state magnetohydrodynamic turbulence. Physics of Plasmas, 18(7):072304, 2011.

[71] Y. Zhou, W. H. Matthaeus, and P. Dmitruk. Colloquium: Magnetohydrodynamic turbulence and time scales in astrophysical and space plasmas. Reviews of Modern Physics, 76(4):1015, 2004.

[72] Ye Zhou. A phenomenological treatment of rotating turbulence. Physics of Fluids, 7(8): 2092-2094, 1995.

[73] F. Zonta, C. Marchioli, and A. Soldati. Direct numerical simulation of turbulent heat transfer modulation in micro-dispersed channel flow. Acta Mechanica, 195(1-4):305-326, 2008. 
[74] F. Zonta, C. Marchioli, and A. Soldati. Modulation of turbulence in forced convection by temperature-dependent viscosity. Journal of Fluid Mechanics, 697:150-174, 2012. 